\title{
Archaeonautica
}

Archaeonautica

L'archéologie maritime et navale de la préhistoire à

l'époque contemporaine

$20 \mid 2018$

De re navali : Pérégrinations nautiques entre Méditerranée et océan Indien

\section{Typologie et influence des bases monoxyles dans la construction navale traditionnelle, à l'image des esquifs réalisés par encorbellement inverse}

Typology and influences of the basic logboat in traditional nautical construction, such as vessels of reverse corbelled construction

\section{Béat Arnold}

\section{OpenEdition \\ Journals}

Édition électronique

URL : http://journals.openedition.org/archaeonautica/345

DOI : 10.4000/archaeonautica.345

ISSN : 2117-6973

Éditeur

CNRS Éditions

\section{Édition imprimée}

Date de publication : 6 décembre 2018

Pagination : 165-182

ISBN : 978-2-271-12263-6

ISSN : 0154-1854

Référence électronique

Béat Arnold, «Typologie et influence des bases monoxyles dans la construction navale traditionnelle, à l'image des esquifs réalisés par encorbellement inverse », Archaeonautica [En ligne], 20 | 2018, mis en ligne le 30 avril 2020, consulté le 30 avril 2020. URL : http://journals.openedition.org/archaeonautica/ 345 ; DOI : https://doi.org/10.4000/archaeonautica.345 


\title{
TYPOLOGIE ET INFLUENCE DES BASES
}

\section{MONOXYLES DANS LA CONSTRUCTION NAVALE TRADITIONNELLE, À L'IMAGE DES ESQUIFS RÉALISÉS PAR ENCORBELLEMENT INVERSE}

\author{
Béat ARNOLD
}

\section{Résumé}

Cette étude a pour objectif une réflexion ou une classification des embarcations basées sur le concept de la soustraction de matière, c'est-à-dire dont l'élément principal est issu de l'évidage d'une volumineuse pièce de bois.

1) La pirogue monoxyle stricto sensu. A l'origine de tout cet ensemble, nous avons la pirogue monoxyle, c'est-à-dire taillée dans un seul tronc. Elle est répartie universellement, là où pousse une matière première ad hoc, c'est-à-dire des arbres suffisamment grands.

2) Base axiale monoxyle. Une autre évolution, tout aussi universelle que l'évidage, réside dans la volonté et la nécessité de construire des embarcations plus grandes que les dimensions offertes par la matière première.

2.1) Surélévation simple (ou extension). Adjonction d'une ou de plusieurs virures sur la base axiale évidée.

2.2) Expansion. L'élément obtenu par soustraction a été déformé plastiquement par chauffage du bois, c'est-à-dire expansé (dans ce dernier cas une surélévation complémentaire est presque toujours présente).

2.3) Balancier(s). Nous avons considéré que l'introduction du balancier correspond, dans les faits, à un développement équivalent à celui de la surélévation ou/et à l'expansion, car elle ouvre des possibilités exceptionnelles pour parcourir les océans, en particulier à l'aide d'une voile, offrant ainsi à l'homme une nouvelle dimension maritime.

2.4) Stabilisateurs. La réduction des dimensions de l'élément axial a occasionnellement induit I'adjonction de stabilisateurs fixés directement contre les flancs de l'élément monoxyle afin d'augmenter la stabilité transversale.

2.5) Solutions de remplacement de la base monoxyle. Exceptionnellement, d'autres solutions ont été développées afin d'approcher la forme d'un monoxyle façonné par soustraction, en particulier I'arrondi des extrémités, par exemple au moyen d'un empilement décalé de planches étroites, comme c'est le cas des patia des côtes de l'Orissa; une technique que nous avons qualifiée de construction par encorbellement inverse.

3) Monoxyles multiples. Un troisième ensemble est constitué par des pirogues couplées (3.1), c'est-à-dire formé par deux monoxyles reliés par une plate-forme. On peut également mentionner les constructions résultant d'une accumulation de pirogues juxtaposées (usuellement entre 2 et 5), dérivant plutôt du concept du radeau (3.2); le principe de soustraction pouvant, dans ce cas, plutôt être considéré comme une solution destinée à obtenir l'un des éléments de flottaison d'une sorte de radeau.

4) Embarcations à éléments monoxyles assemblés. Un dernier ensemble est formé, à l'origine, par des pirogues monoxyles fendues selon leur axe longitudinal en deux valves, entre lesquelles ont été insérées des planches. Afin de dépasser les dimensions imposées par la matière première, chacune des valves a, par la suite, été façonnée par réduction dans deux ou trois troncs. Ces éléments ont directement induit la forme de l'embarcation qui peut être inscrite dans un polygone simple. Trois problèmes fondamentaux ont alors dû être résolus: assembler les divers éléments longitudinaux, assurer une rigidité transversale suffisante (mais également longitudinale pour les plus grandes embarcations), rendre les coutures, c'est-à-dire la coque, étanche.

\section{Mots clés}

Balancier, Base axiale monoxyle, Construction par encorbellement inverse, Monoxyle assemblée, Monoxyle expansée, Monoxyle multiple, Monoxyle surélevée, Orissa, Patia, Pirogue monoxyle, Radeau, Stabilisateur

TYPOLOGY AND INFLUENCES OF THE BASIC LOGBOAT IN TRADITIONAL NAUTICAL CONSTRUCTION, SUCH AS VESSELS OF REVERSE CORBELLED CONSTRUCTION

\section{Abstract}

This study intends to consider and classify those craft that are based upon the idea of the removal of matter, that is, where the principal element is the result of hollowing a voluminous piece of wood.

1) The logboat or monoxylous boat. The origin of this ensemble is the logboat, which has been carved from a single trunk. It is to be found everywhere the raw material, i.e. sufficiently large trees, grows.

2) Monoxylous axial base. Another evolution, just as widespread as hollowing, arises from the need to build craft that are bigger than the dimensions of the raw material.

2.1) Extension. The addition of one or more strakes to the hollowed out axial base.

2.2) Expansion. The element obtained by extraction is shaped by heating the wood, that is, it is expanded (in this case there is nearly always an increase in the height of the craft through additional elements).

2.3) Outrigger(s). We consider that the introduction of an outrigger is effectively an equivalent development to increased height and/ or expansion since it opens up the possibility of oceangoing travel, in particular with the aid of a sail, and thus offers humans a novel maritime dimension.

2.4) Stabilisers. The reduction of the dimensions of the axial element has sometimes led to the addition of stabilisers attached directly onto the flanks of the monoxylous element in order to increase transversal stability. 
2.5) Solutions for the replacement of the monoxylous base. Other solutions have been developed in order to approximate the shape of a logboat made by removal of matter, in particular the rounded extremities, for example by staggered piling of thin planks, as in the case of the patia of the Orissa coasts; we have called this technique reverse corbelled construction.

3) Multiple-log boats. A third ensemble is composed of paired logboats (3.1), that is, made of two logboats connected by a platform. One can also mention constructions resulting from a number of logboats set next to each other (usually between 2 and 5) that derive rather from the concept of a raft (3.2); the principle of removal of matter can in this case be better considered as a solution designed to obtain a flotation element for some kind of raft.
4) Craft of assembled monoxylous elements. A final ensemble is made from logboats that have been split along their length into two halves of a shell into which planks are inserted. In order to extend the dimensions imposed by the raw material, each of the halves has thereafter been cut into two or three timbers. These elements directly impose the shape of the craft, which can be described as a simple polygon. Three basic problems must then be solved: the assembly of the different longitudinal elements; assurance of sufficient transversal rigidity (and also longitudinal for the largest craft); rendering the seams, and thus the hull, watertight.

\section{Key words}

Outrigger, Monoxylous axial base, Reverse corbelled construction, Extended logboat, Expanded logboat, Multiple-log boat, Raised logboat, Orissa, Patia, Logboat, Raft, Stabiliser
Nous dédions cet essai à notre ami et collègue Patrice Pomey, qui s'intéresse lui aussi aux embarcations traditionnelles (Pomey 2012), afin de mieux comprendre les vestiges archéologiques ou la mise en œuvre de certaines techniques, telle la ligature et la couture des bordages ${ }^{1}$.

Ce travail résulte directement des observations que nous avons pu réaliser lors de nos pérégrinations en Afrique, en Asie ou en Amérique du Sud (Arnold 2011), où l'embarcation de base est souvent constituée par une pirogue monoxyle. Or le façonnage de ces dernières devient de plus en plus problématique en raison d'une exploitation à outrance des forêts, en particulier des essences les plus grandes, induisant un coût prohibitif pour les pêcheurs de cette matière première exportée vers les pays occidentaux. Des paramètres qui obligent les indigènes à mettre en œuvre des solutions adaptées et souvent originales afin de pouvoir poursuivre leurs activités. Ainsi, des convergences peuvent parfois être observées avec le mobilier archéologique.

C'est également l'occasion d'aborder la problématique posée par la spécificité des pirogues à balancier(s) parcourant les eaux du Pacifique et de l'océan Indien, ou les découvertes récentes de grands chalands gallo-romains en Europe continentale, mais également d'évoquer les étranges patia utilisés par les pêcheurs de l'Orissa.

\section{POUR UNE CLASSIFICATION PREMIÈRE DES EMBARCATIONS TRADITIONNELLES ARCHAÏQUES}

Plus qu'un long discours, les plans publiés en 1930 par Hans Suder, indiquant l'emplacement des occurrences d'embarcations en peau ou en écorce, de radeaux simples ou évolués, de pirogues ou d'autres embarcations, soulignent l'aspect universel de la répartition de ces divers types de structures flottantes en fonction du milieu environnemental (fig. 1). Ils excluent par là même toute approche diffusionniste, même si à une échelle régionale ou suprarégionale un tel phénomène doit parfois être pris en considération, au même titre que des transferts de technologie. Ils soulignent également que, pour les formes les plus proches de la matière première brute, la transformation de cette dernière et les méthodes d'assemblages sont en nombre fini,

1. Nous remercions notre collègue Eric Rieth pour la relecture de cet article et ses observations, et Maeva Arnold pour la mise au net de la figure 20. relevant l'importance des études ethnographiques et archéoethnographiques.

Les cartes de répartition de $\mathrm{H}$. Suder ont notablement influencé les auteurs germaniques (Lächler, Wirtz 1962, p. 196, 208 et 214; Spranz (éd.) 1984, p. 9-12; etc.). Parallèlement, il classe les embarcations en «Behelfsmittel», à savoir les «schwimmende Körper», où le corps de l'homme est en contact direct avec l'eau, et les «Fahrzeuge » comprenant les «Flösse» (c'est-à-dire les radeaux constitués par un assemblage de «Behelfsmittel») et les bateaux (Suder 1930, p. 6). Il souligne également que les esquifs sont directement dépendants des matériaux à disposition avec un recours privilégié au bois, lorsque ce dernier est présent. Il classe ensuite ces esquifs en «Holzfahrzeuge» et «nicht aus Holz hergestellte Fahrzeuge», ces derniers étant également subdivisés en radeaux et en embarcations (ibid. p. 6 et 91).

Dans ce contexte, nous devons évoquer le travail novateur de Julien Le Roy (1777), probablement influencé par la publication des récits des nombreuses circumnavigations accompagnés d'illustrations diffusées à très large échelle, par exemple par Theodor de Bry (et ses successeurs) dans son édition en 14 volumes des Grands Voyages (entre 1590 et 1634), puis des Petits Voyages en 13 volumes (entre 1597 et 1628; Camus 1802). Comme l'a relevé Lucien Basch (1987, p. 23-25), Le Roy compare l'évolution de la construction navale antique (Le Roy 1777, p. 12-15, pl. 1), en s'appuyant sur les textes d'Homère, Hérodote, Pline et Quintilien, aux radeaux de roseaux des Erythréens (au sud de la mer Rouge), aux esquifs constitués par une canne fendue utilisée par les indigènes des Indes ${ }^{2}$ et aux «monoxyles» issus d'un «arbre creusé» (ibid. p. 22), tout en évoquant les barques constituées par une armature de «bois plians recouverts de cuir ou de papyrus » (ibid. p. 22). Les deux premières planches de son ouvrage sont, par leur forte similitude, très évocatrices de cette problématique.

Les travaux et multiples publications de James Hornell, synthétisés ou plus précisément réunis dans sa monographie Water transport, origins and early evolution (1946), sont le résultat direct de ses inlassables observations réalisées sur le terrain pendant plus de quatre décennies (Hornell 1946, p. vii-viii), en particulier en Inde, en Extrême-Orient et dans le Pacifique ${ }^{3}$. Son travail, largement diffusé, a eu un impact majeur sur les

2. C'est-à-dire d'une tige d'un grand roseau. Dans ce contexte, il ne peut guère s'agir que d'une vision chimérique du bambou.

3. Pour ce dernier espace, on se reportera aux trois monographies de Haddon et Hornell 1936-1938. 

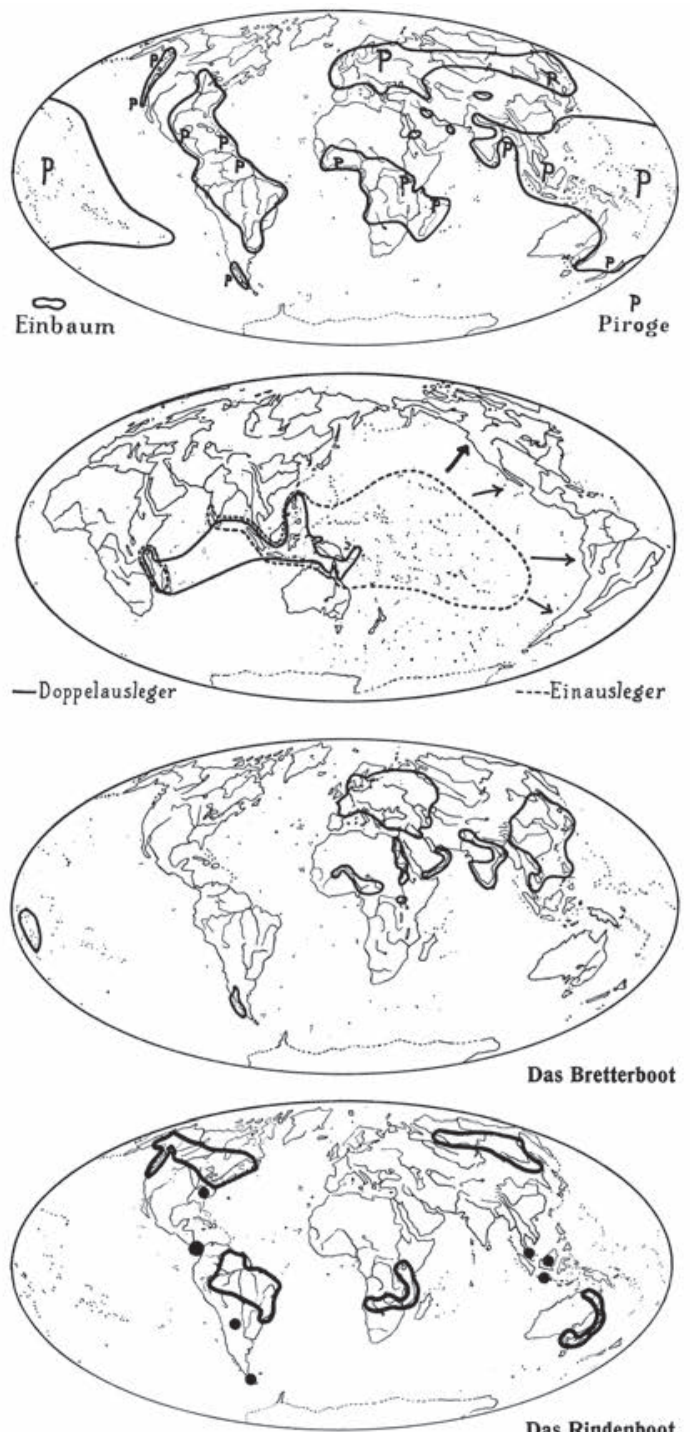

Das Rindenboot
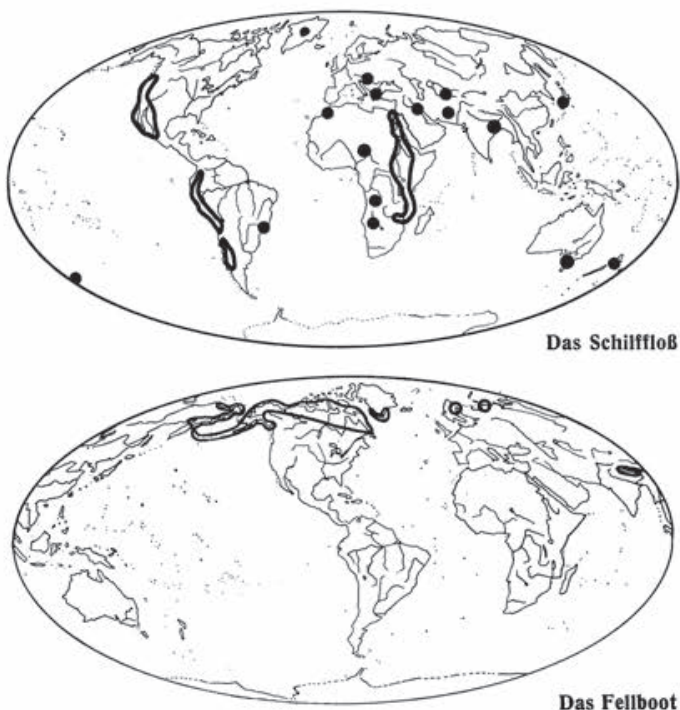

Fig. 1: Répartition des différents types de structures flottantes (6 des 14 plans présentés dans Suder 1930): monoxyle et esquif surélevé/expansé; balancier double et simple; barque à planches; esquif en écorce; radeau en roseau; esquif en peau. recherches en ethnologie maritime. Sans développer une typologie à proprement parler, la structure même de ses chapitres en constitue indirectement une au travers de ses «groups»: A «floats, rafts and kindered craft», B «skin boats», C «bark canoes, dugouts and plank-built craft». Les «floats» sont subdivisés (ibid. p. 1), à l'image de l'étude de H. Studer, en «swimming floats» et «riding floats» où, dans le premier ensemble, l'homme est en contact direct avec l'eau. La juxtaposition de plusieurs de ces éléments constitue le second ensemble où l'homme s'affranchit plus ou moins du milieu aqueux en se hissant sur la structure flottante. Il conclut l'analyse de son premier «group» en recherchant, pour les jonques, une origine dans la pirogue-double (ibid. p. 88) ou plus probablement dans les radeaux de bambous ou de poutres (ibid. p. 90 ; voir également Poujade 1946, p. 245-249).

Pour son «group C», J. Hornell (1946, p. 188) propose très clairement comme origine des pirogues monoxyles des embarcations façonnées dans une seule écorce, brute (ibid. p. 182183), à l'image de celles observées, par exemple, sur la rivière Murray en Australie (Edwards 1972). Il affirme que l'abattage de grands arbres et leur évidage avec « the rudest of stone tools » (Hornell 1946, p. 181) n'a eu lieu que beaucoup plus tardivement, même en prenant en considération une utilisation intensive du feu. Dans ce contexte, on peut souligner que le plus ancien canoë en écorce date de 940-810 av. J.-C. (Ua-42695, 2735 \pm 35 BP), et fut découvert en 1934 à Byslätt (Horred, Västergötland; Lindberg 2012).

J. Hornell considère que le type le plus primitif de monoxyle est constitué par un demi-cylindre dont les deux extrémités sont fermées par des matières rapportées. Nous suivrons plutôt sa seconde proposition avec des monoxyles aux extrémités tronquées et taillées obliquement, et plus précisément en cuillère, une forme qui peut être en partie obtenue directement lors de l'abattage du tronc, même à l'aide du feu (Hariot 1590, respectivement pl. XIII et XII). En effet, obtenir une surface plane, verticale ou oblique, est toujours le résultat d'un travail conséquent où le recours à des haches munies d'un tranchant métallique au fil étroit est quasi indispensable. Il en va évidemment tout autrement avec le développement de la grande scie en fer (scie passe-partout) où l'obtention d'une tranche verticale correspond à un travail minimal. La phase suivante consiste à surélever les flancs de la base monoxyle en y ligaturant des bordages et en y ajoutant une membrure. Dans une étape ultérieure, la base monoxyle peut être expansée, élargie en la chauffant, à l'image de la pirogue néolithique de Paris/Bercy 6 (4810-4360 av. J.-C. ; fig. 2).

Dans son ouvrage de 1945, Jacques Bidault décrit, après une esquisse des découvertes archéologiques et de celles issues de l'Antiquité, d'innombrables esquifs traditionnels en les groupant, à l'image du travail de H. Suder, en «radeaux, outres, canots en vannerie, pirogues de peau, pirogues d'écorce, pirogues monoxyles, pirogues de tronc élargi, doubles pirogues, pirogues à balancier et pirogues de planches ». Albert Eskeröd (1956) présente une approche similaire, mais exclusivement axée sur des découvertes réalisées en Scandinavie, en essayant finalement de montrer que les embarcations à clin proto-vikings ne dérivent pas des embarcations en peau. Enfin, il classe les pirogues monoxyles en «hard dugouts» et, pour celles expansées, en «soft dugouts with inserted frame system».

Dans sa monographie de 1976, Basil Greenhill développe une classification plus formelle (1976, p. 91) et propose de subdiviser les esquifs qui ne sont pas constitués de planches 


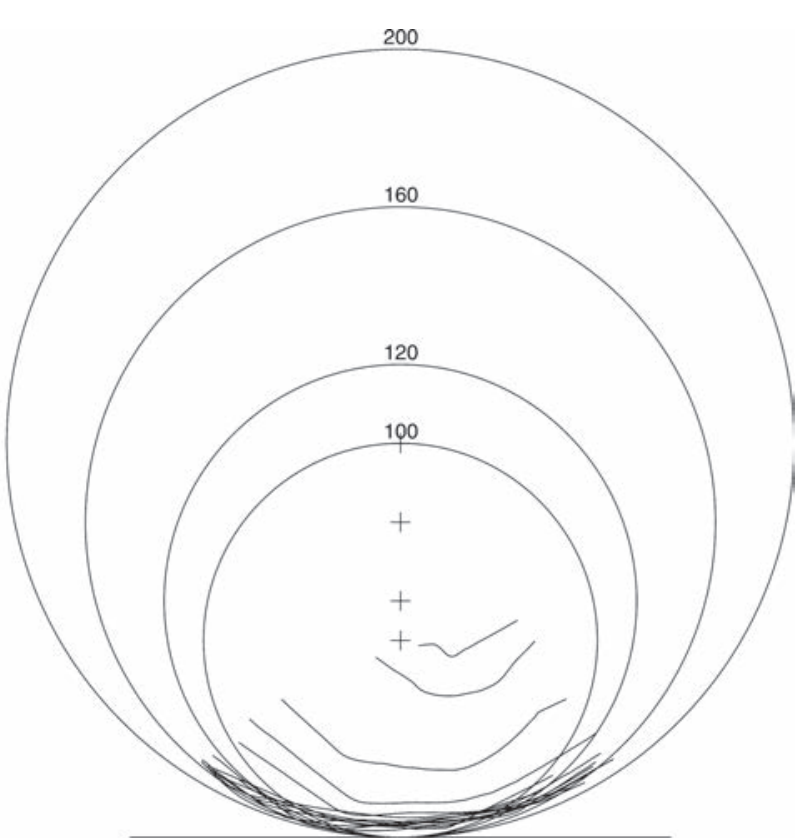

Fig. 2: La pirogue du début du Néolithique moyen (groupe de Cerny) de Paris-Bercy 6 présente, vers son extrémité conservée, une moelle de l'arbre située à $50 \mathrm{~cm}$ au-dessus du fond. Dans la partie médiane, la moelle devrait se situer à 90-100 cm au-dessus du fond, si l'on reconstitue son emplacement par géométrie, un fait qui témoigne d'une expansion de cette coque en chêne épaisse de $3 \mathrm{~cm}$ (Arnold 2006, p. 66, fig. 2).

assemblées en quatre «roots », ou ensembles originels. Ces derniers ont pu évoluer de manière indépendante, mais ont également pu être influencés ultérieurement par les embarcations composées de planches. Il s'agit de l'ensemble radeau, bateau en peau, esquif en écorce et pirogue, ce dernier étant initialement constitué par des spécimens obtenus par l'évidage d'un tronc. Dans son analyse de ces quatre «roots », B. Greenhill intègre de multiples embarcations à planches. Ainsi, il associe aux «rafts and raft boats» les embarcations à fond plat (ibid. p. 97) et les embarcations chinoises du type jonque (ibid. p. 100), en suivant dans ce dernier cas l'approche proposée par J. Hornell. Il évoque également le radeau constitué de faisceaux de papyrus ligaturés qui est à l'origine des embarcations à planches présentes sur le Nil à l'époque des pharaons (ibid. p. 106). Enfin, il inclut dans cet ensemble les découvertes de l'âge du Bronze de North Ferriby et celle plus tardive de Brigg, découvertes dans la Humber (ibid. p. 111), embarcations que nous aurions plutôt vu être classées dans le «root» des pirogues.

Le quatrième ensemble est constitué à l'origine par les monoxyles, dont la lignée est «extremly complexe, starting and stopping again at different times in different parts of the world» (ibid. p. 129). B. Greenhill ne développera toutefois pas cet aspect. Parmi les éléments marquant l'évolution de cet ensemble, il évoque l'expansion de l'élément monoxyle, l'extension par l'adjonction d'une virure ou fargue afin d'augmenter l'importance du franc-bord, voire de plusieurs virures (ibid. p. 134). Il incorpore dans ce groupe les embarcations scandinaves à clin en se référant à l'approche élaborée par Ole Crumlin-Pedersen (1972). Enfin, l'adjonction de balanciers permet d'augmenter ou plutôt d'assurer une stabilité transversale aux esquifs munis d'une coque étroite et haute. Parfois la base monoxyle se réduit notablement tout en servant d'assise à une ou plusieurs virures (Greenhill 1976, p. 138), en suivant ainsi l'analyse développée par Wolfgang Rudolph (1966, p. 9-10) dans le cadre de son

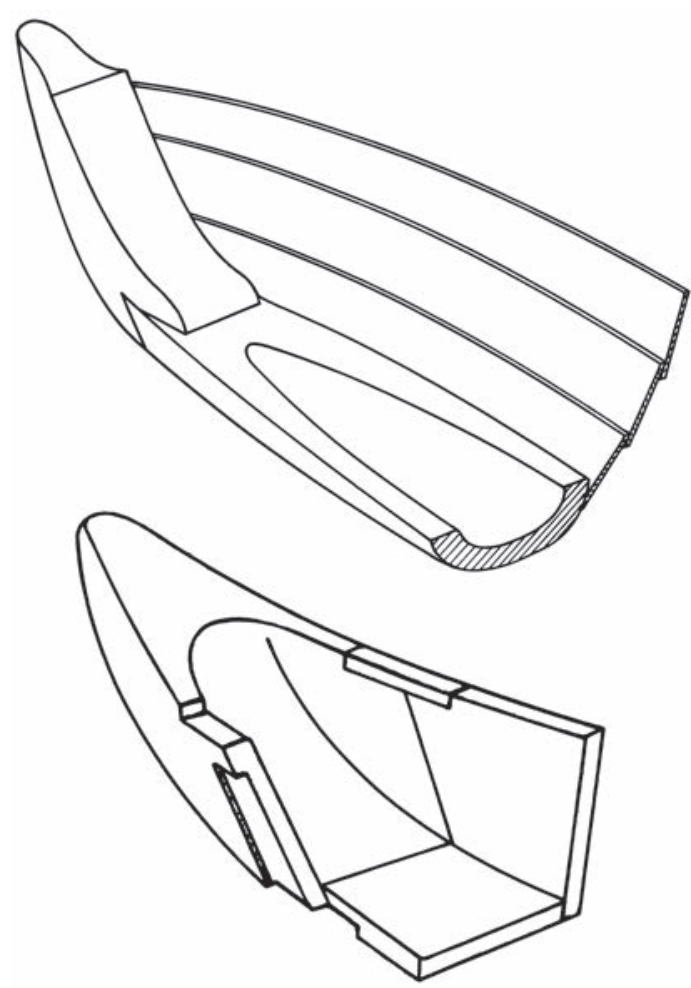

Fig. 3: Vue isométrique d'un Bodenschalenboot (Kahn) de Rostock (à gauche), avec sa base évidée massive (dernier vestige du monoxyle originel), et d'un Blockstevenboot (Spitzkahn) de Gratz sur l'Oder, avec son étrave/étambot évidé dérivé du monoxyle (Rudolph 1966, fig. 6 et 27, pl. 2 et 5).

étude sur les embarcations vernaculaires de Poméranie. Ce dernier utilise une classification décimale, où il y a d'un côté les monoxyles et de l'autre les embarcations à planches, ellesmêmes subdivisées en: 1) les «Bodenschalenboote», dont la base est constituée d'un élément monoxyle massif partiellement évidé (par exemple le Kahn de Rostock; fig. 3 haut); 2) celles dont le fond est constitué par une ou plusieurs planches longitudinales; 3) celles munies de courtes planches transversales; 4) les bateaux à quille. Dans le groupe 2, une attention particulière est portée à la pièce ou aux éléments constituant les extrémités; pièces parfois très massives obtenues par soustraction de matière dans une portion de tronc, à l'image des techniques utilisées dans le façonnage des monoxyles (fig. 3 bas). Par la suite, Seán McGrail (1985; 1987, p. 4-11, fig. 2.2) proposera une classification dichotomique incorporant l'ensemble des structures flottantes et même des types dont on ne connaît actuellement pas encore d'exemplaire. On notera ainsi le rôle stratégique joué par la pirogue monoxyle dans toutes ces approches ${ }^{4}$.

Notre propos sera plus sectoriel, et spécifiquement centré sur la famille des embarcations dont la ou les parties essentielles sont basées sur le concept de soustraction de matière ${ }^{5}$, c'est-àdire le façonnage d'une volumineuse pièce de bois, dont l'élément le plus symbolique est illustré par la pirogue monoxyle.

4. On peut également mentionner, par exemple, les travaux de Halldin 1950, p. 82-89; Christensen 1977; Rieth 1979 et 1981; Arnold 1992, 13, p. 57-75; Medas 1993.

5. Sur la problématique entre les principes de conception (formes et structures) et les procédés de construction, c'est-à-dire les moyens techniques mis en œuvre, on se reportera aux travaux de Patrice Pomey $(1988,1994,1998,2004)$ et d'Éric Rieth (2006, p. 63-65). 

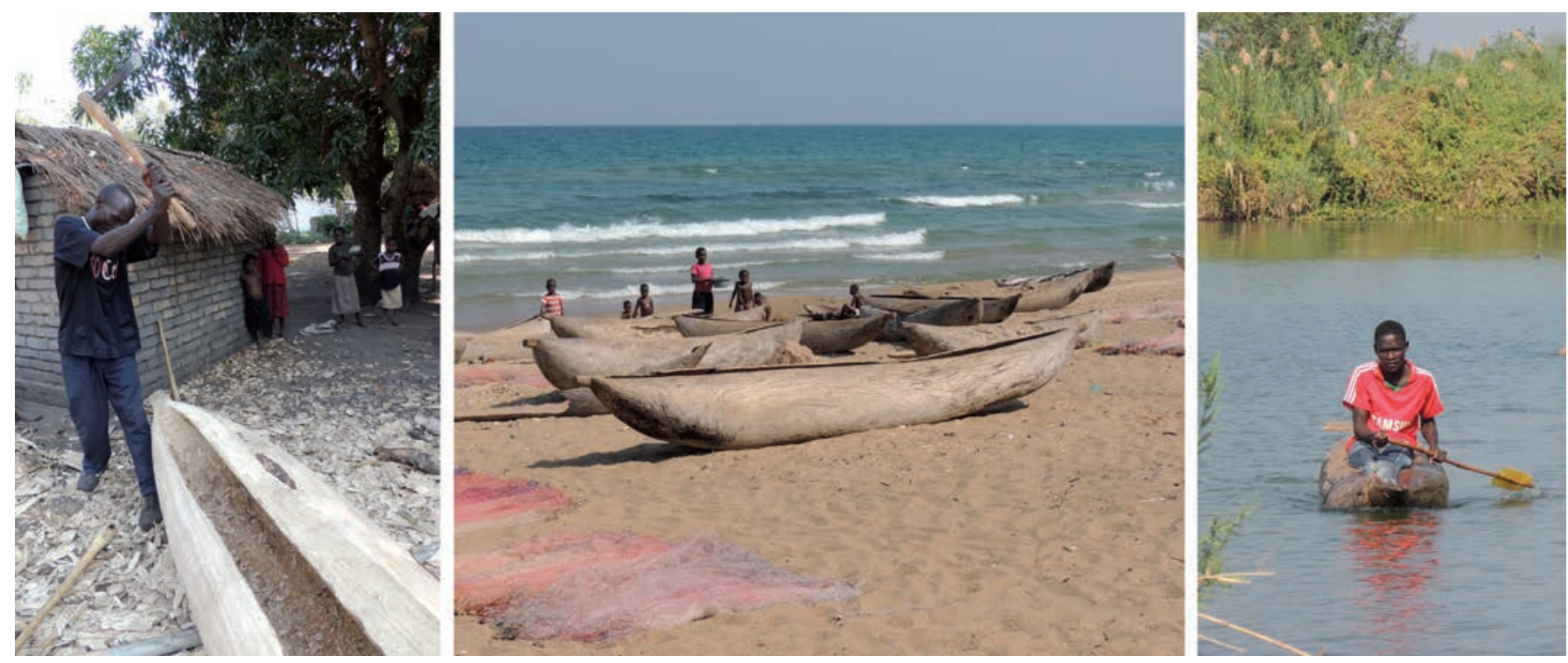

Fig. 4: Bwato du lac Malawi: la saignée médiane n'est pas destinée à permettre une expansion des flancs; les bords resserrés servent simplement de bancs longitudinaux, et l'ensemble ne pose pas de problèmes de façonnage au charpentier spécialisé (clichés B. Arnold ; Sanga vers Nkhata Bay et Chia Lagon, Malawi ; juillet 2012).

\section{PIROGUE ET MATIÈRE PREMIÈRE}

Le cadre est donc dressé, et nous nous pencherons pour commencer sur les pirogues monoxyles et les problèmes qui ont été induits lorsque les dimensions de la matière première n'ont plus été suffisantes.

Les moyens pour se déplacer sur l'eau sont basés sur le principe d'Archimède et ils peuvent être subdivisés en deux grands ensembles: soit c'est la densité réduite d'une masse compacte, généralement constituée d'éléments assemblés, qui dégage la possibilité de supporter une charge (par exemple les radeaux), que l'homme soit partiellement immergé ou non, soit c'est une enveloppe creuse qui procure une portance importante, tant et aussi longtemps que cette dernière ne se remplit pas d'eau.

L'un et l'autre peuvent d'ailleurs être combinés, comme l'illustrent les milliers de pirogues actuellement utilisées sur le lac Malawi (ou Nyassa). Elles sont façonnées dans un bois tendre et léger où, en cas de tempête ou de naufrage, la masse de bois constituée par le volume du fond, épais, et des flancs permet au pêcheur de s'accrocher à un objet flottant lui assurant sa survie. Il s'agit du gmelina (Gmelina arborea), dont la densité est de l'ordre de 0,4-0,6. Cette essence à croissance très rapide a été introduite des Indes afin de compenser le déboisement (Mills 1994; Louppe (éd.) 2008, p. 329-334). En effet, on estime que 40 années de croissance sont suffisantes pour qu'un arbre puisse être converti en une pirogue dont la longueur atteint 3,5-4 m. Or ce bois tendre n'offre qu'un spectre d'utilisation très réduit: il ne peut, en particulier, pas être utilisé pour les charpentes. C'est donc la convergence de facteurs exogènes ${ }^{6}$, induisant la présence en abondance d'un nouveau matériau, qui a permis le maintien de la tradition du monoxyle dans cette région.

Ces pirogues, appelées $b_{w a t o}{ }^{7}$, sont caractérisées par la présence d'une étroite saignée longitudinale, de largeur constante

6. Lutte contre le déboisement et l'érosion des sols, importation d'une essence à croissance rapide mais dont les qualités mécaniques sont des plus réduites.

7. Ce qui veut dire pirogue en chichewa.
(17-20 cm), permettant d'évider le tronc (fig. 4). Fréquemment un tel processus est mis en œuvre lorsqu'on cherche à écarter artificiellement les flancs d'un monoxyle en l'expansant, afin de quitter la géométrie imposée par la matière première (la forme du fût de l'arbre) et augmenter la stabilité transversale. Tel n'est pas le cas des bwato où cette saignée joue un rôle fondamental dans l'utilisation de l'esquif. Vu son étroitesse, le centre de gravité du piroguier est toujours situé au-dessus de celui de la masse de bois. Le pêcheur peut également travailler debout, pour mettre à l'eau des filets ou les retirer (même avec un lac formé), en coinçant ses mollets contre le sommet des flancs. Pour pagayer, il pose ses fesses sur le sommet de chacun des flancs. Si deux piroguiers sont présents, ils s'asseyent de biais, chacun sur un flanc différent. Pour les anciens esquifs encore taillés dans des bois durs, plus longs (5-6 m) que les spécimens actuels (3,1-4,5 m), les pêcheurs se répartissent le long de l'esquif en fonction de leur nombre. Le sommet incurvé des flancs officie ainsi comme bancs longitudinaux.

Dans le cas des Blockkähne de Poméranie évoqués ci-dessus, la diminution de l'élément basal obtenu par réduction, par façonnage d'une volumineuse pièce de bois, a induit la nécessité d'ajouter une planche pour constituer chaque flanc et, afin de relier ces pièces et celle du fond, de tailler chaque extrémité dans un volume important de bois à l'image des extrémités d'un monoxyle (fig. 3). Pour des embarcations plus grandes, deux voies peuvent être envisagées pour une base axiale. La première consiste à ajouter un nombre plus important de virures, quitte à laisser l'élément axial très massif et qui officiera parfois comme un patin lors de la mise à l'eau du bateau ou lorsqu'il sera à nouveau hissé sur la berge. On parlera de pirogues surélevées (Poujade 1946, p. 186), ou de pirogues avec une surélévation simple.

À Bali, nous avons constaté que la diminution récente des dimensions des arbres destinés au façonnage des pirogues à balancier au corps élancé, les jukung (à ne pas confondre avec ceux du bassin du Barito qui seront évoqués ci-dessous), a obligé les charpentiers à modifier leurs stratégies en réalisant des pirogues surélevées. Comme nous avons pu le constater en discutant avec divers artisans, leur problème n'est pas situé dans 

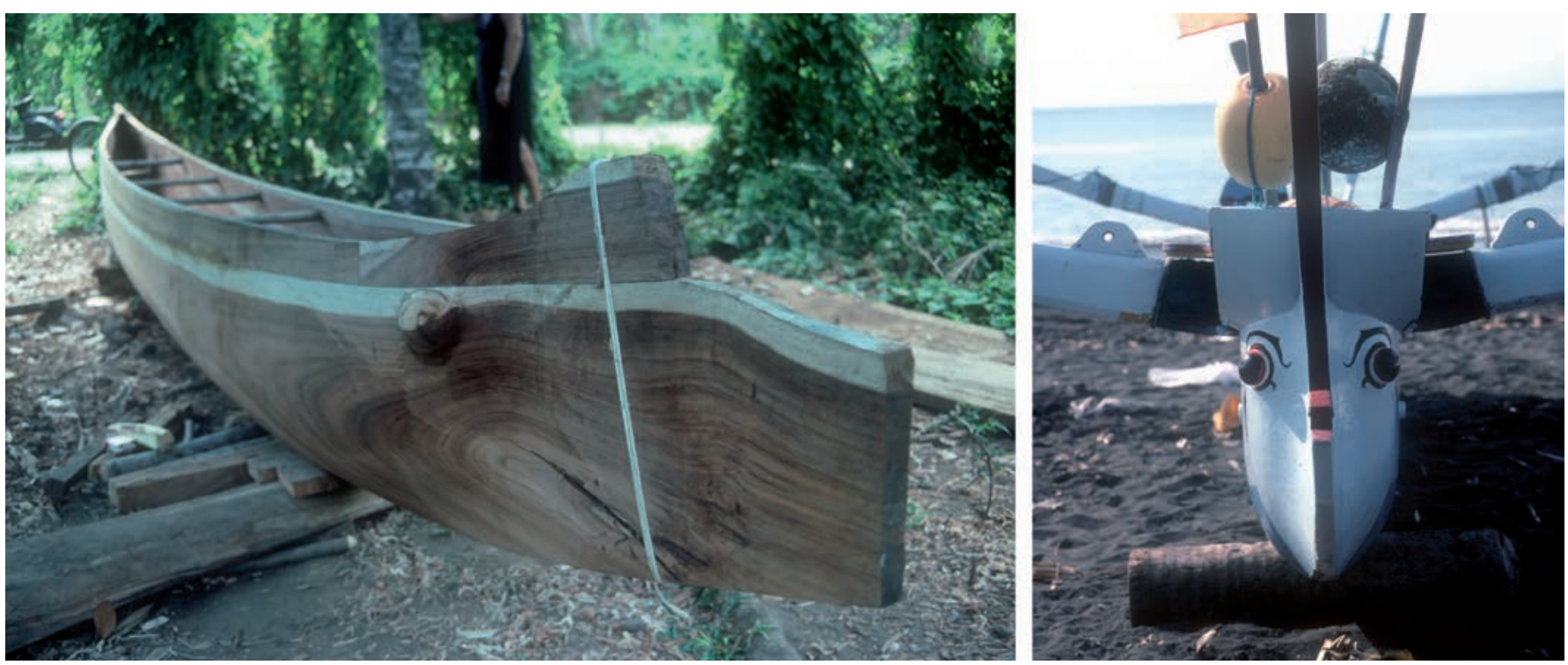

Fig. 5: Jukung de Bali en cours de construction: les dimensions du tronc étant actuellement souvent trop faibles, la base a dû être surmontée par quelques pièces de bois rapportées. Les deux occlus proéminents ont, toutefois, encore pu être réservés dans la masse monoxyle (clichés B. Arnold ; Padang Bai, Bali, Indonésie; octobre 2002).
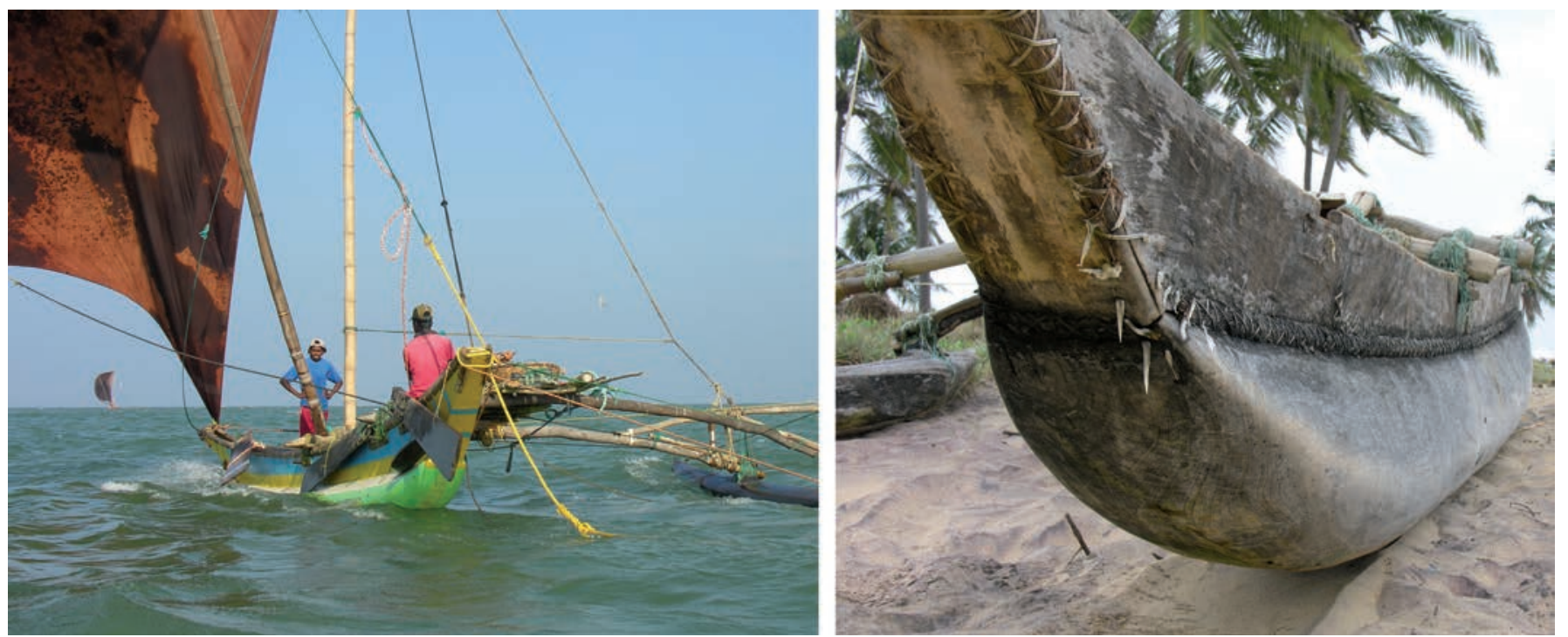

Fig. 6: Pirogue du type oru, avec un balancier et une voile, utilisée ici pour pêcher la crevette à l'aide d'un chalut tiré grâce au vent; la base est maintenant constituée par un élément en fibre de verre de forme identique aux anciens spécimens monoxyles (clichés B. Arnold ; Negombo, Sri Lanka ; janvier 2011).

l'adjonction d'une ou de plusieurs pièces de bois dans la partie haute de la coque (un surcroît du temps de travail est sans importance pour eux), mais de pouvoir donner à l'esquif sa forme effilée qu'il est quasiment impossible d'obtenir au moyen de planches assemblées (fig. 5). Une forme que l'on ne peut guère atteindre autrement qu'en recourant à de nouvelles technologies: celles du moule et de la fibre de verre.

Un tel processus s'est généralisé au Sri Lanka pour faire face à la destruction quasi totale de sa flotte vernaculaire par le tsunami de décembre 2004, au sud de Colombo, qui a également emporté d'innombrables pêcheurs et constructeurs de pirogues $^{8}$. L'aide internationale s'est traduite par l'apport dans de

8. Dans ce contexte, la monographie de Gerhard Kapitän (2009) tient, hélas, dorénavant plus de l'ethnoarchéologie que de l'ethnographie. multiples villages de la technologie de la fibre de verre, la seule à pouvoir répondre à la reconstruction de toute la flottille en un temps minimal (ou acceptable). L'absence d'une centralisation d'une telle production a toutefois permis de garder la diversité des formes locales et des techniques de pêche (fig. 6, partie gauche). En revanche la construction traditionnelle de bateaux en bois, aux bordages ligaturés, a totalement disparu. Ainsi, il ne nous a été donné de n'observer plus que trois embarcations de ce type encore en usage en janvier 2011. Au nord de Colombo, la situation n'est pas plus brillante (même en l'absence des effets destructeurs du tsunami): les anciennes embarcations vernaculaires ont été hissées sur le haut des plages et sont à l'abandon (voir fig. 15), progressivement remplacées par des spécimens en fibre de verre dont la technologie a été acquise au travers des ateliers œuvrant au sud (fig. 6, partie droite).

Une seconde voie consiste à obtenir un élément axial plus 

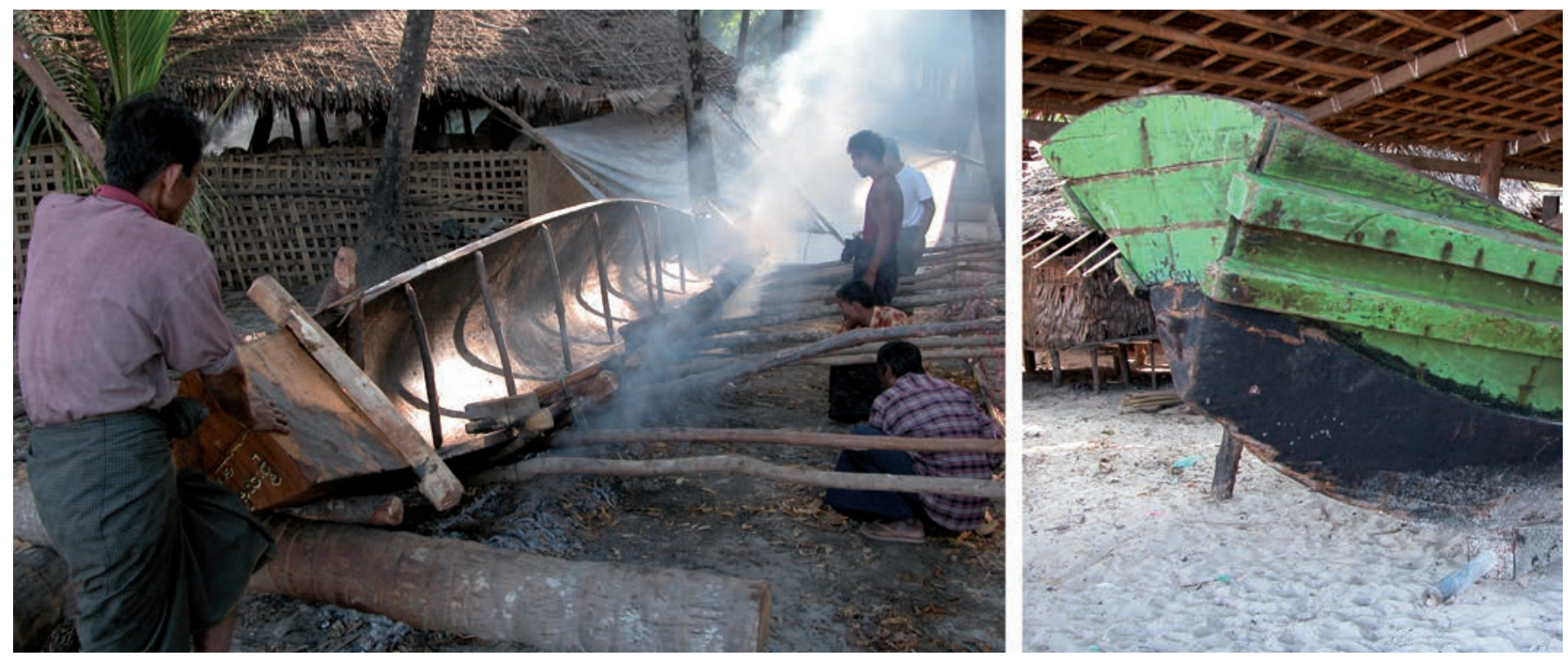

Fig. 7: Grande barque de pêche motorisée se'hlay de la côte ouest du Myanmar, dont la base est constituée par un élément monoxyle expansé au feu (clichés B. Arnold ; Lone Tha, au sud-ouest de Thandwe, Myanmar ; janvier 2005).
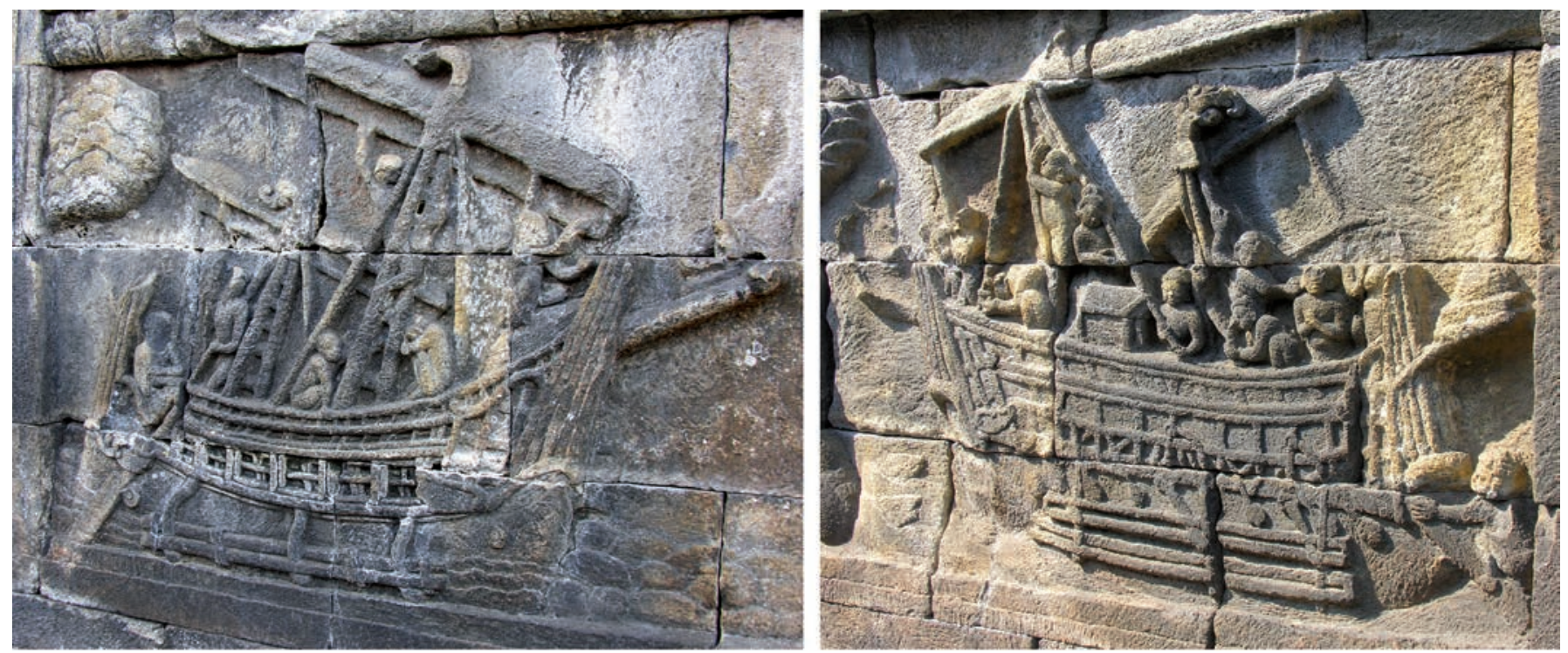

Fig. 8: Deux des bas-reliefs présents sur la première galerie du stupa de Borobudur, illustrant des voiliers à balanciers, munis de deux mâts. Les prises de vue obliques de la proue de l'un d'entre eux (à gauche) et de la poupe d'un autre soulignent la présence d'extrémités tout en rondeur, témoins d'une base monoxyle très probablement expansée (clichés B. Arnold ; Java ; août 2009).

grand que le fût original de l'arbre, tout en donnant à l'esquif une section très évasée. Le principe consiste à écarter artificiellement les parois en chauffant le bois. On parlera alors de pirogues expansées. Cette expansion va induire un abaissement encore plus marqué du franc-bord au maître-bau, entraînant dans la plupart des cas l'adjonction d'une planche (ou fargue) sur les flancs, voire de plusieurs virures, donc une surélévation des flancs. Le bateau du début du $\mathrm{XI}^{\mathrm{e}}$ siècle découvert à Utrecht en 1930, par exemple, est de ce type, et l'analyse des cernes de croissance permet de mettre en évidence cette technique avec un déplacement géométrique important de la moelle dans la partie centrale de l'embarcation (Van de Moortel 2003, fig. 28.5-7). Cette technique est encore fréquemment utilisée pour la fabrication de nombreux bateaux de grandes dimensions, par exemple en Asie du Sud-Est, tels les divers jukung du bassin du Barito, à Bornéo (Petersen 2000) ou les grands bateaux de pêche se'hlay de la côte ouest du Myanmar (fig. 7). Dans un tel contexte, tout laisse à penser que les voiliers à balanciers et à deux mâts (en tous cas ceux de dimensions moyennes) gravés sur les parois des galeries du stupa géant de Borobudur (érigé entre 850 et 950 sur l'île de Java) ont une coque dont l'essentiel est constitué par une vaste base monoxyle probablement expansée (fig. 8).

En ce qui concerne la pirogue de Paris/Bercy 6 (début du Néolithique moyen), évoquée précédemment, le but premier a probablement consisté à obtenir une pièce exceptionnellement légère afin de pouvoir la transporter d'un plan d'eau à un autre, et tout laisse à penser que ses flancs n'ont pas dû être surélevés. 

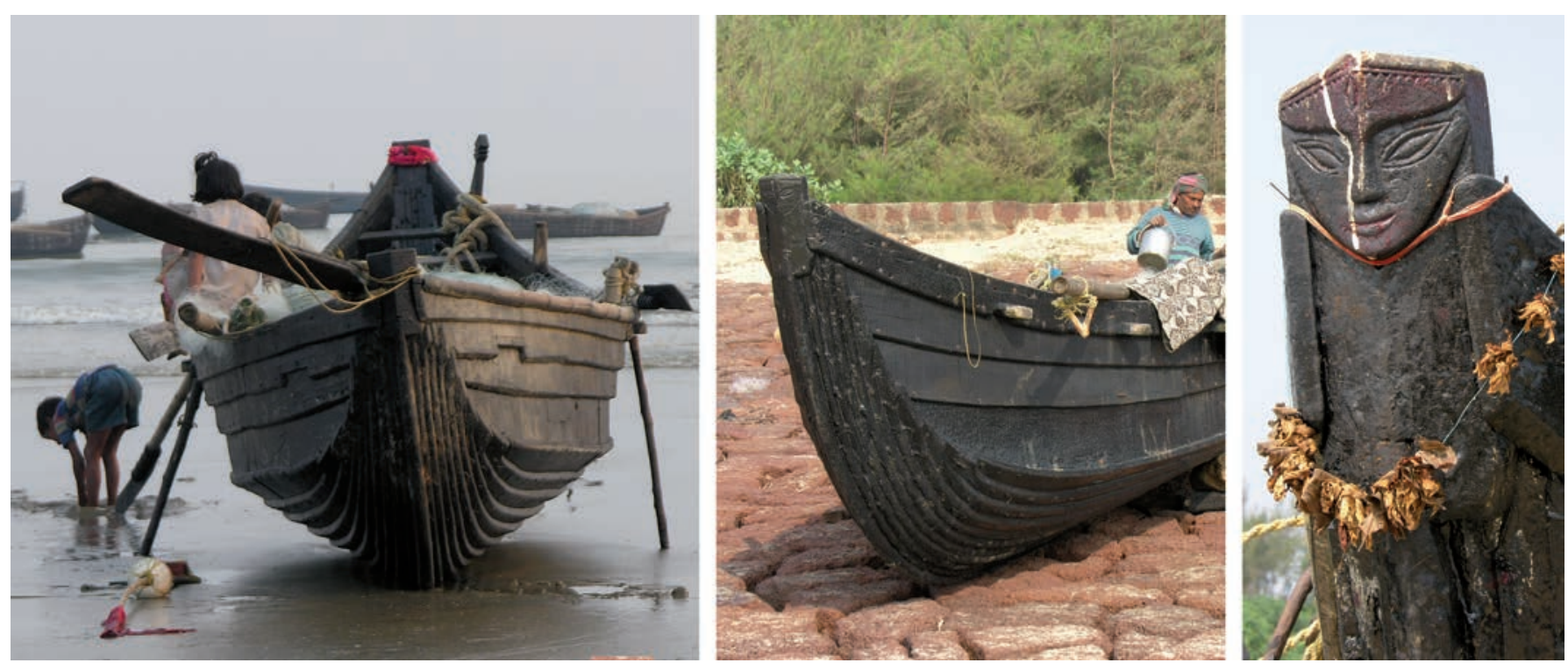

Fig. 9: Petite barque de pêche appelée patia, utilisée sur la côte nord de l'Orissa, entre Kasafal et Digha. Sa base est constituée par un empilement de planches qualifié de construction par encorbellement inverse, dont la fonction est probablement de se substituer à une base monoxyle. A gauche, gros plan sur la poupe; à droite, proue surmontée d'une représentation de la déesse Kali (clichés B. Arnold ; flanc ouest de Digha, Indes ; novembre 2008).

\section{LES PATIA ET LA CONSTRUCTION PAR ENCORBELLEMENT INVERSE}

Remplacer les bases monoxyles, en raison de la disparition progressive des arbres séculaires ou de l'impossibilité d'avoir accès à ce type de matériau, a inévitablement induit la nécessité de substituer le façonnage par réduction par d'autres solutions, par exemple par un assemblage d'éléments longitudinaux, tout en sachant que la disposition de ces derniers aux extrémités rend problématique le façonnage à l'identique de l'arrondi des extrémités d'un monoxyle.

Dans ce contexte, une petite barque de pêche utilisée sur la côte nord de l'Orissa a retenu notre attention par la discontinuité structurelle entre le fond et les flancs, soulignée par un bouchain vif. Il s'agit des patia (fig. 9). Ces derniers ont fait récemment l'objet d'une étude approfondie (Blue et al. 1997; McGrail 2003, p. 72-97). Les plus petites embarcations (longueur 6,5-8,5 m) sont propulsées à la voile et à l'aide de rames. Elles sont hissées sur le haut des plages après chaque sortie en mer. Des spécimens plus grands (8,5-10,5 m), équipés d'un moteur inbord, sont stationnés dans les estuaires.

La coque est constituée par deux types de planches bien distinctes: d'une part celles constituant la structure du fond, très étroites, d'autre part celles formant les flancs, très larges. Les planches étroites sont empilées les unes sur les autres avec un léger décalage vers l'extérieur, ouvrant lentement la largeur de la coque. La pièce la plus basse, la plus longue, est arquée aux extrémités et dessine le profil longitudinal de l'esquif. Les dernières virures étroites présentent une légère inclinaison vers l'intérieur. Cet ensemble, en forme de banane, donne à la base de la coque un aspect massif qui est comparé à une sorte de colonne vertébrale (Blue et al. 1997, p. 198). Cet empilement de planches, en particulier en y associant les dernières virures légèrement inclinées, a été décrit comme un assemblage à clin inversé (ibid. p. 194-195). Les larges virures constituant les flancs sont en revanche clairement disposées à clin.

Le «high-rising reverse-clinker planking» de la partie basale

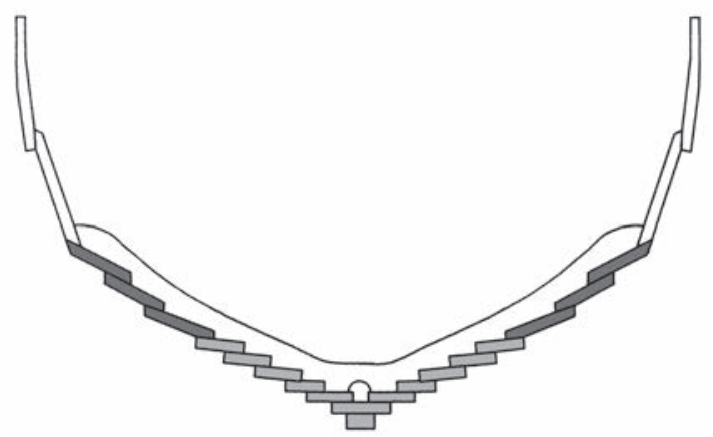

Fig. 10: Coupe d'un patia au maître-bau: en gris, les éléments constituant la construction par encorbellement inverse (d'après un relevé de Blue et al. 1997, fig. 7).

des patia est considéré comme une innovation du $\mathrm{XIX}^{\mathrm{e}}$ ou du $\mathrm{XX}^{\mathrm{e}}$ siècle (McGrail 2003, p. 96), en le mettant en parallèle avec le peettoo $a^{9}$, ou avec les esquifs utilisés sur les rivières situées en amont de Sylhet, au Bangladesh (ibid. p. 25-66): des arguments qui militent, en fait, plutôt en faveur d'une autre origine pour la partie basale des patia. Nous pensons que cet agencement particulier des planches étroites du fond correspond à une solution particulière visant à trouver un moyen pour remplacer une base monoxyle façonnée tout en rondeur (fig. 10). Le procédé employé n'est pas sans rappeler la construction des toitures en pierre de nombreux bâtiments monumentaux, où chaque assise de dalles ou de blocs repose en grande partie sur la couche inférieure qu'elle ne dépasse que légèrement, une technique appelée construction par encorbellement. Ainsi, rangée après rangée l'ouverture du toit se referme progressivement. Sommes-nous dans le cas des patia en présence d'une adaptation d'un tel mode de construction, ou pas? Il ne nous est

9. Voir par exemple le dessin réalisé par Balthazar Solvyns à la fin du XVIII ${ }^{\mathrm{e}}$ siècle (Hardgrave 2004, p. 478). 

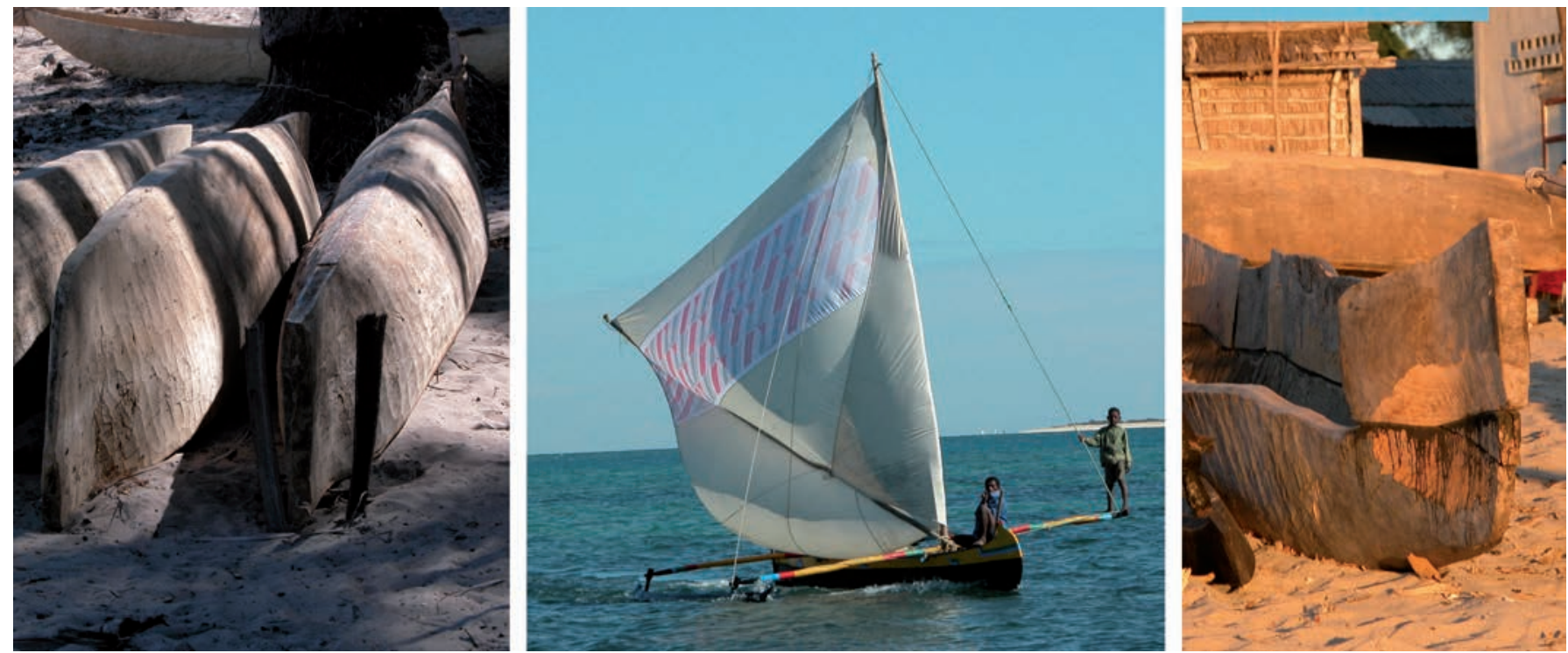

Fig. 11: Pirogues du type laka fihara munies d'un balancier: à gauche, profil effilé de la base monoxyle; à droite, la rareté des grandes billes oblige maintenant de plus en plus les charpentiers à façonner par réduction des pièces complémentaires complexes destinées à surélever la base monoxyle, puis de les fixer au moyen de chevilles en bois chassées dans les cans (clichés B. Arnold; Anakao, Tuléar et Belo-sur-Mer, côte ouest de Madagascar ; juin-juillet 2003).

évidemment pas possible de conclure avec certitude, mais une convergence des solutions mises en œuvre semble évidente ${ }^{10}$. Nous nous proposons donc de qualifier la base des patia comme une construction par encorbellement inverse.

\section{PIROGUES À BALANCIER(S)}

Aux deux types de pirogues évoluées présentées ci-dessus (surélevées et expansées), il nous faut ajouter les pirogues à balancier(s), esquifs particulièrement fréquents dans l'océan Indien et dans le Pacifique ${ }^{11}$. En effet l'adjonction de balancier(s) induit un nouveau type d'esquif (dont la base monoxyle est fréquemment surélevée, voire parfois même expansée). Pour de nombreux spécimens, il n'est souvent pas possible d'utiliser la coque toute seule en raison de l'instabilité initiale de l'élément monoxyle trop effilé, trop étroit (fig. 5): ce dernier, dépourvu de son/ses balancier(s), se couche ainsi instantanément sur l'un de ses flancs, en raison de son métacentre situé en dessous du centre de gravité. L'introduction d'un balancier va également favoriser l'utilisation d'une voile et ouvrir l'espace de navigation en augmentant notablement les distances parcourues quotidiennement, c'est-à-dire en offrant l'océan aux utilisateurs de ces esquifs.

10. Pour l'Orissa on peut, par exemple, mentionner le temple de Sûrya à Konârak, construit au XIII ${ }^{\mathrm{e}}$ siècle en bord de mer et abandonné au XVII ${ }^{\mathrm{e}}$ siècle; à Puri, on a le célèbre temple hindouiste de Jagannath du XII ${ }^{\mathrm{e}}$ siècle (Groslier 1982). Ces dates laisseraient à penser que l'origine de la construction basale des patia pourrait être notablement antérieure au XIX ${ }^{\mathrm{e}}$ siècle.

11. La présence de balancier(s) n'est pas connue en Europe. Les trois mortaises quadrangulaires présentes dans la pirogue de l'âge du Bronze de ChalainMarigny, découverte en 1904 et exposée au musée de Lons-le-Saunier, avaien été interprétées comme des éléments en relation avec un balancier (Grandsaignes 1906). Une analyse détaillée que nous avons pu effectuer sur les traces de travail présentes dans ces mortaises, caractérisées par l'absence d'une décomposition de ces traces dans la zone médiane, montre que ces évidements sont contemporains de la découverte et de l'extraction de la pirogue des sédiments encaissants (Arnold 1996, p. 115).

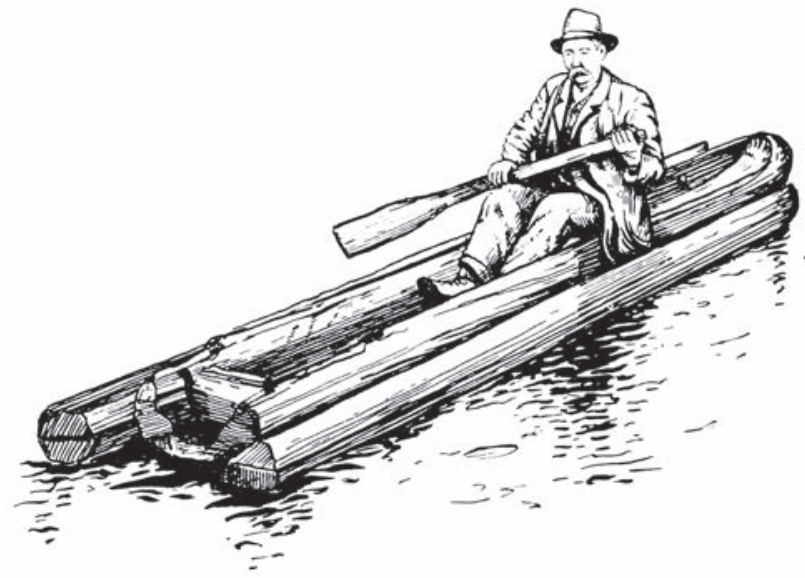

Fig. 12: Petite pirogue munie de stabilisateurs (Värmland, Suède ; Eskeröd 1956, fig. 7B)

Dans ce contexte, on relèvera que la perte d'un tel élément, surtout s'il est unique, est la cause de multiples accidents mortels, comme nous avons hélas pu le constater à Madagascar (fig. 11).

Ainsi, nous pensons que les pirogues à balancier(s) doivent être considérées comme un ensemble spécifique à part entière, au même titre que les pirogues surélevées et celles expansées, en raison des changements fondamentaux qu'a induits la présence de ce dispositif.

\section{PIROGUES MUNIES DE STABILISATEURS}

Un autre groupe de pirogues est caractérisé par la présence d'un élément axial de faibles dimensions, dont la stabilité transversale a été améliorée en y accolant un flotteur contre chaque flanc, au niveau de la flottaison (fig. 12), voire, s'il est vraiment très petit, en l'incorporant dans une véritable ceinture (Itkonen 1941, p. VIII, fig. 23-24 et 87; Eskeröd 1956, p. 63-65). 
Contrairement à l'adjonction de balanciers (très écartés du corps central), les stabilisateurs correspondent plutôt à une solution de fortune pour pallier la dimension réduite de la base monoxyle, sans ouvrir de nouvelles perspectives pour l'utilisation de ces esquifs et sans impact pour une quelconque évolution des constructions navales. On observe essentiellement sa présence dans des espaces où l'utilisation des balanciers est inconnue. La Scandinavie est l'une des régions où l'on a observé de nombreux esquifs de ce genre, mais également la côte ouest de l'Amérique du Sud (Lothrop 1932, p. 230-231).

\section{PIROGUES COUPLÉES ET PIROGUES JUXTAPOSÉES}

Un autre ensemble structurel particulier est constitué par les pirogues couplées ${ }^{12}$. Ces dernières sont formées par deux éléments, constitués chacun par une pièce monoxyle, souvent rehaussée, voire expansée (Petersen 2000, p. 68-69), réunis par deux barres de liaison, ou parfois beaucoup plus. L'ensemble est généralement surmonté par une plateforme augmentant notablement la surface utile (fig. 13), constituant une autre entité aux performances spécifiques. Dans les eaux intérieures, elles officient souvent comme bacs permettant le transport d'une rive à l'autre de cargaisons volumineuses, tel un chariot, un groupe de personnes ou du bétail. On peut ainsi mentionner près d'une centaine de découvertes d'éléments monoxyles de ce type dans les rivières du sud de l'Allemagne, datant du Moyen Age, dont certaines possèdent encore les vestiges de l'une des barres de liaison (Ellmers 1973, Kröger 2009 et 2011). Sur la Save, qui se jette dans le Danube à Belgrade, des pirogues couplées sont souvent ancrées dans le cours d'eau et soutiennent un moulin (Brunner 1903, p. 11-12) ${ }^{13}$.

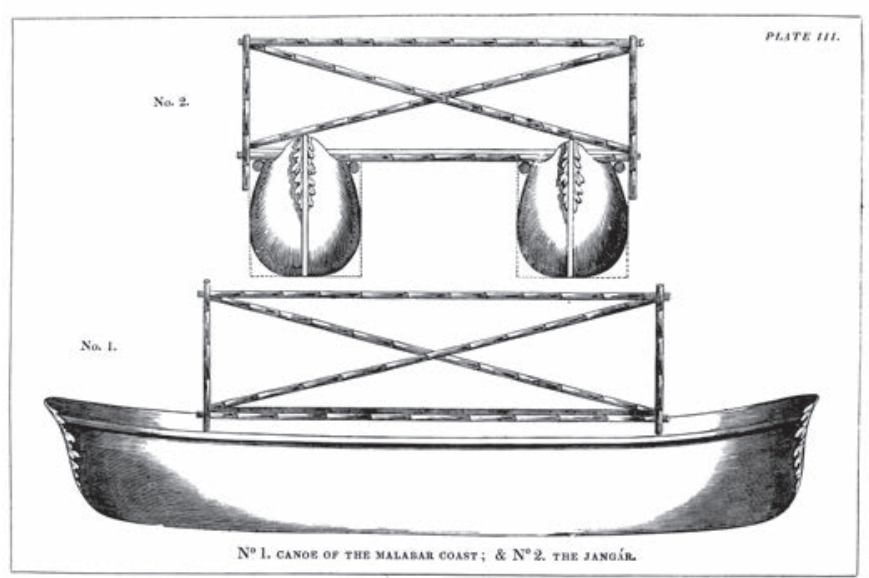

Fig. 13: Pirogue couplée munie d'une plateforme entourée de garde-fous (jangár de la Côte de Malabar ; Edye 1834, pl. 3).

12. Nous préférons ce terme à celui de catamaran en raison de l'emploi de ce dernier pour des radeaux élaborés utilisés le long des côtes de l'Inde et du Sri Lanka (en fait les kattu-maran; c'est-à-dire «poutres attachées ensemble» en tamoul); et actuellement de certains voiliers de course.

13. Voir également Gräf 2006, p. 118-119, 144-147 et 171-172.
Dans l'océan Pacifique, elles ont parfois atteint des dimensions exceptionnelles, comme en a rencontré le capitaine James Cook lors de son deuxième voyage et dont il a fait dresser les plans (Cook, Furneaux 1777, vol. 1, pl. XV et XVI). Elles ont joué un rôle essentiel dans la colonisation des îles du Pacifique.

La rivière Dunajec, en Pologne, présente un cas singulier où des monoxyles autonomes (au nombre de cinq sur les clichés publiés par Boczar 1966) sont reliés entre eux afin de constituer une structure flottante. Cette accumulation de pirogues juxtaposées s'apparente plutôt, dans son concept, à celui du radeau. Nous intégrons aussi dans un tel ensemble les paires de pirogues accolées, même si leurs extrémités présentent parfois une dissymétrie selon l'axe longitudinal (Itkonen 1941, fig. 34-35).

Pirogues couplées et pirogues juxtaposées font partie, d'un point de vue conceptuel, de l'ensemble des embarcations à multiples éléments. Dans la problématique qui nous intéresse ici, le recours à la soustraction de matière pourrait être considéré comme une solution technique, utilisée pour la confection des divers éléments de la structure flottante. A moins que les découvertes archéologiques ne permettent de montrer, dans le futur, qu'à l'origine des esquifs couplés il n'y avait que des flotteurs obtenus par soustraction, ceux formés par des embarcations en planches, à fond plat ou à quille n'étant apparus que secondairement. Dans ce dernier cas de figure, ce sont les embarcations à planches qui joueraient alors le rôle de solution technique destinée à remplacer les éléments monoxyles.

\section{ASSEMBLER, RENDRE ÉTANCHE ET RIGIDIFIER}

Pour les charpentiers voulant dépasser les contraintes imposées par les dimensions de la matière première aux pirogues monoxyles, trois problèmes fondamentaux devaient être résolus : relier des pièces de bois entre elles, assurer l'étanchéité au niveau des coutures, assurer une rigidité transversale (et plus tard longitudinale) suffisante. Quant aux éléments intermédiaires insérés dans le fond ou de surélévation constituant les flancs, ils ont, parfois, également été obtenus par réduction.

De manière quasi universelle, le mode d'assemblage de différents éléments a été la ligature et la couture avec, pour les liens, les paramètres sensibles constitués par leur résistance mécanique, leur élasticité et leur durabilité. La zone critique est située au niveau de la résistance relative du bois enserré par les liens, et au niveau de ces derniers, en raison des tensions induites dans la coque lors de la navigation. En plaquant sur la couture un bourrelet, une masse fibreuse pour assurer l'étanchéité, cette dernière officie également comme amortisseur, à tel point que, parfois, les trous destinés au passage des liens fixant les virures supérieures entre elles ne sont même pas rendus étanches par l'insertion de petites chevilles, seule la fonction d'amortisseur étant jugée nécessaire (fig. 14, en haut à gauche). Les traces les plus anciennes de ligatures ont été observées sur des pirogues du Mésolithique final, au Danemark (vers 5260-4790 av. J.-C.; Andersen 1994).

On constate parfois le recours à une autre méthode basée sur l'utilisation de pièces de bois de liaisons: tenons et/ou chevilles. Ils trahissent l'existence d'une société où l'art de la charpenterie est très développé. En présence d'une combinaison chevilles et/ou tenons, le problème de l'étanchéité se pose en d'autres termes. En général il est résolu en posant un matériau ad hoc au moment de l'assemblage des deux pièces de bois à joindre (principe du lutage). 

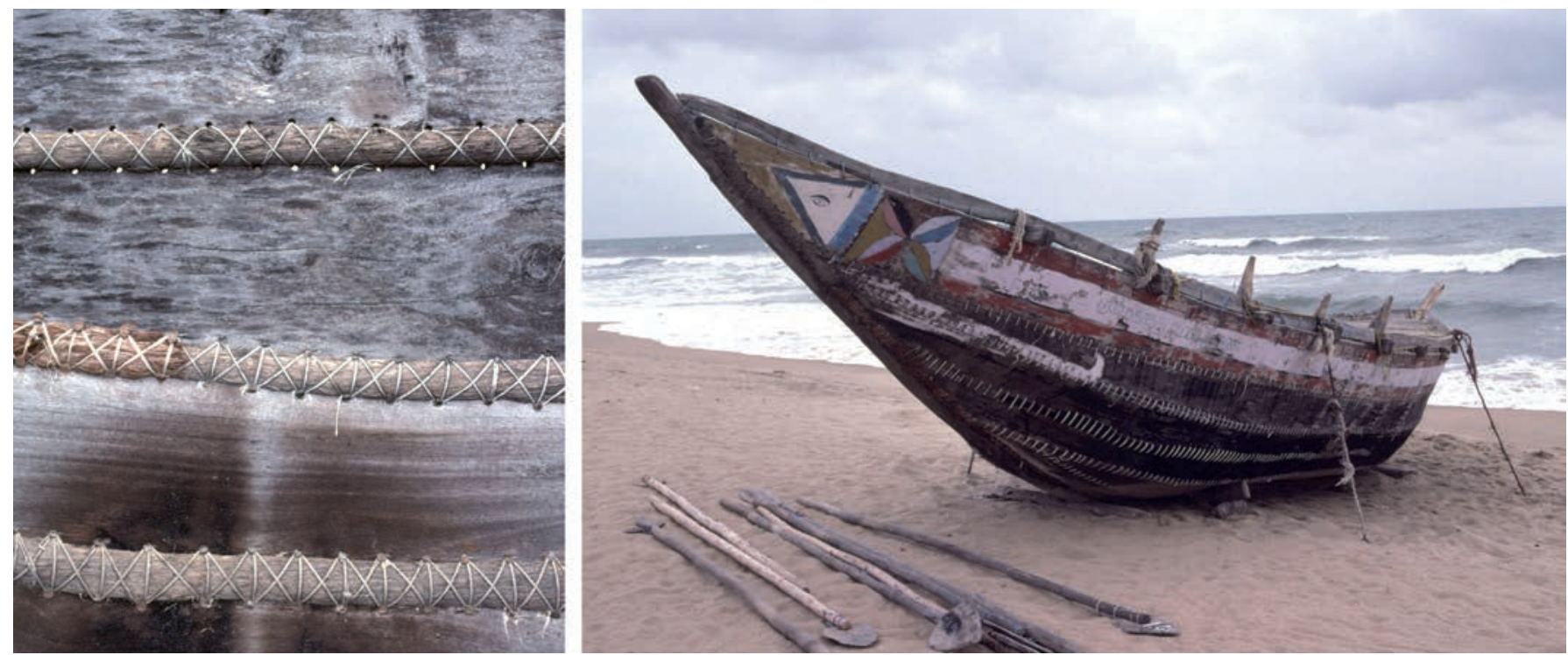

Fig. 14: Partie supérieure du flanc d'un masula, où le bourrelet d'étanchéité sur une couture (ici celle située au sommet) ne sert parfois plus que d'amortisseur pour les ligatures (clichés B. Arnold ; Tirupporur, au sud de Madras, Indes ; février 2000).

La rigidité transversale semble avoir été plus problématique à résoudre, comme l'illustrent les embarcations de l'âge du Bronze découvertes en Angleterre, à North Ferriby et plus récemment à Douvres, où des clés de section modeste sont chassées au travers de reliefs mortaisés de grandes dimensions réservés à la surface des «planches» constituant le bordé, pièces obtenues par réduction afin d'y façonner ces reliefs mortaisés. Un tel dispositif pose des problèmes notables lorsqu'il s'agit de réparer des clés brisées, et encore plus des reliefs. Une évolution pragmatique a consisté à poser les éléments transversaux, la membrure, au-dessus des reliefs devenus plus réduits et percés d'une petite mortaise permettant juste le passage de la ligature, afin de fixer la membrure, à l'image des embarcations scandinaves de l'âge du Fer de Hjortsping ou pré-viking de Nydam, par exemple (Crumlin-Pedersen 2010), une technique également en usage au $\mathrm{xx}^{\mathrm{e}}$ siècle en Extrême-Orient, les ligatures pouvant être aisément réparées (Horridge 1982).

\section{LES EMBARCATIONS POLYGONALES}

Aux pirogues monoxyles et à celles caractérisées par la présence d'un élément axial monoxyle, c'est-à-dire façonné par réduction, on peut opposer un autre ensemble où la «partie» monoxyle est formée par deux éléments situés au niveau du bouchain, entre lesquels sont généralement insérées des planches. Ces dernières peuvent, parfois, être également façonnées par réduction. Le concept de base peut, en quelque sorte, être illustré par une pirogue monoxyle fendue selon son axe longitudinal en deux valves (Itkonen 1941, p. IX). Ces dernières présentent donc une section transversale en forme de $\mathrm{L}$ ou parfois de $\mathrm{C}$ et assurent, en raison de ce profil, une grande partie de la rigidité longitudinale.

Les embarcations gallo-romaines découvertes dans l'espace rhodanien et rhénan appartiennent pour l'essentiel à ce groupe. Dans le cas d'Yverdon-2 (Arnold 1992, vol. 2, p. 27), chacun des éléments en $\mathrm{L}$ a été taillé par réduction dans un tronc. En écartant ces éléments, il est possible d'élargir le bateau en y insérant des planches, à l'image des $m \bar{a}$-däl-pāru utilisés au Sri Lanka (fig. 15; Kapitän 2009). Si les extrémités de l'embarcation se referment, l'extrémité des virures posées sur les bouchains monoxyles devra également être façonnée par réduction afin de se plaquer, de s'intégrer dans la forme arrondie de l'extrémité considérée (fig. 16). Si l'on veut obtenir des embarcations dont la longueur dépasse les 12-15 m, chaque valve devra être remplacée par deux pièces, ou bordés monoxyles de transition, mises bout à bout. Pour celles de plus de 20-22 m, ce sont trois pièces qui seront nécessaires. Ces dernières, taillées dans un tronc, sont donc rectilignes et vont influencer directement la forme générale de l'embarcation dont le fond pourra s'inscrire dans un polygone simple (fig. 17). L'une des évolutions de ces embarcations polygonales, qualifiées par Eric Rieth (1981, p. 56) de «monoxyles assemblés $»^{14}$, verra la partie verticale de la pièce en $\mathrm{L}$ se réduire de plus en plus pour finalement n'avoir comme fonction que d'offrir une masse de bois suffisante pour clouer le premier bordage des flancs (Vliermann 1996, p. 104), constitué dans le cas des chalands du groupe 1 de Lyon/Parc Saint-Georges (épaves 2, 3, 4, 5 et 7) par un énorme demi-tronc de sapin (Guyon et Rieth 2001, p. 96).

Plus l'embarcation sera grande, plus les problèmes posés par la rigidité longitudinale seront importants, en particulier si l'une des parties du bouchain monoxyle est réduite, à l'image des chalands gallo-romains du type 1 de Lyon/Parc Saint-Georges évoqués ci-dessus. Pour ces derniers, la rigidité longitudinale repose indubitablement, pour ne pas dire exclusivement sur les bordages de flanc en demi-tronc. On peut mentionner le cas remarquable du chaland gallo-romain de Xanten-Lüttingen, long de plus de $30 \mathrm{~m}$, qui présente non seulement un bouchain monoxyle mais également, sur le haut des flancs, une pièce massive taillée par réduction en forme de gouttière (fig. 18). Plus généralement, le clouage d'une large planche contre la face extérieure du haut des flancs constitue une solution fréquemment utilisée. C'est par

14. Nous préférons le concept «à éléments monoxyles assemblés », que nous emploierons d'ailleurs pour qualifier un ensemble plus large d'embarcations, à savoir les embarcations polygonales, celles constituées par un patchwork d'éléments monoxyles et les pirogues aboutées (cf. infra). 

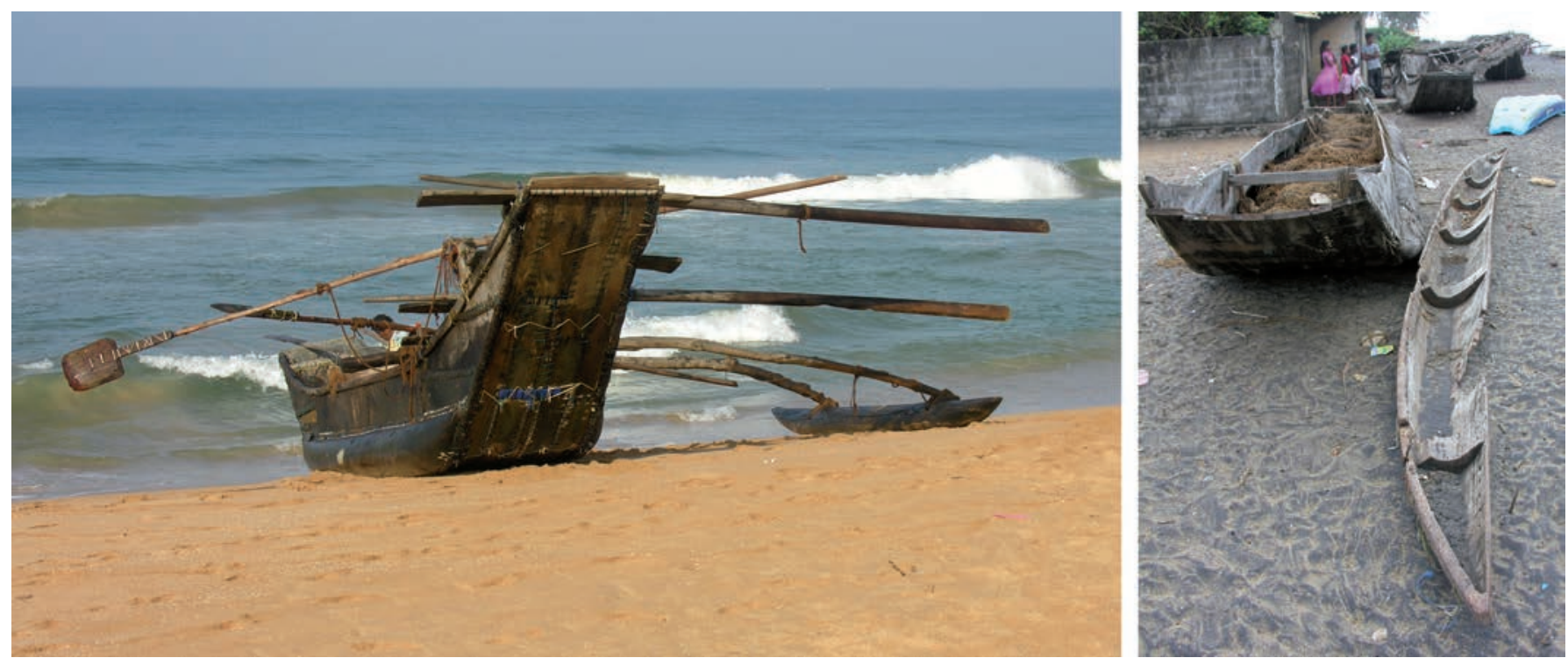

Fig. 15: Les bouchains des mā-däl-pāru sont fortement taillés par réduction dans un tronc, jusqu'à prendre une forme de $C$ souvent renforcée par quelques nervures transversales (voir le bouchain détaché à droite). En écartant ces éléments, il est possible d'élargir considérablement le bateau en y insérant des planches (clichés B. Arnold; Bentota à gauche et Negombo à droite, Sri Lanka ; janvier 2011).

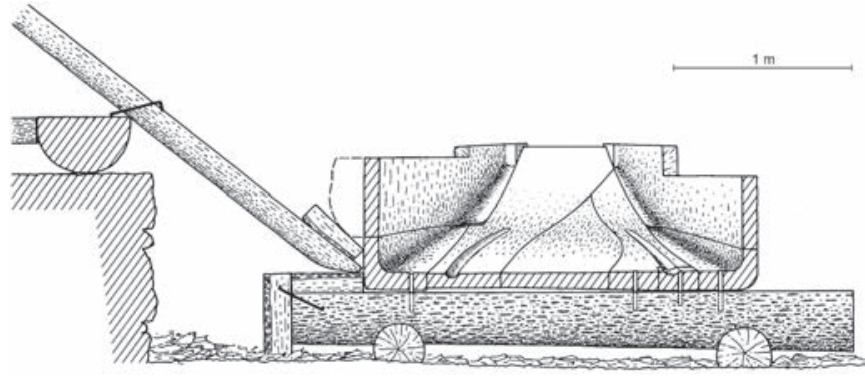

Fig. 16: Coupe transversale illustrant l'installation d'une demi-grume (à gauche, en traits discontinus) sur le bordage monoxyle de transition et son façonnage par réduction en cuillère (Altaripa, réplique du chaland gallo-romain de Bevaix; Arnold 1999, p. 132).

la pose d'un tel élément que le profil en long en forme de W d'Altaripa (réplique du chaland gallo-romain de Bevaix), résultant de la pose du mât et de la rame-gouvernail, a pu être corrigé. Durant les essais de navigation, nous avons constaté que la rame-gouvernail fonctionnait en réalité comme un véritable gouvernail de forme très allongée ${ }^{15}$, avec deux points d'articulation et manœuvré par une barre franche.

Si la pièce constituant le bouchain est remplacée par deux planches, comme c'est le cas des embarcations gallo-romaines du type 2 de Lyon/Parc Saint-Georges (épave 8), la géométrie de l'embarcation peut quitter le carcan des polygones et décrire une forme elliptique. Ce procédé se généralisera d'ailleurs de plus en plus avec le développement du sciage de long pour l'obtention des planches. Une technique qui ne pourra évidemment être mise en œuvre que parallèlement à une maîtrise du forgeage de grandes lames en fer (ou à leur importation).

On obtient ainsi les trois grandes traditions d'architecture nautique fluviale mises en relief par E. Rieth (2006, p. 26-30):

15. Ce manche très allongé se termine au-dessus de la tête du pilote, à l'image des stèles gallo-romaines de Cologne, de la déesse Nehalennia ou du batelier Blussus (Arnold 1992, 2, p. 57, 84a, 85e)

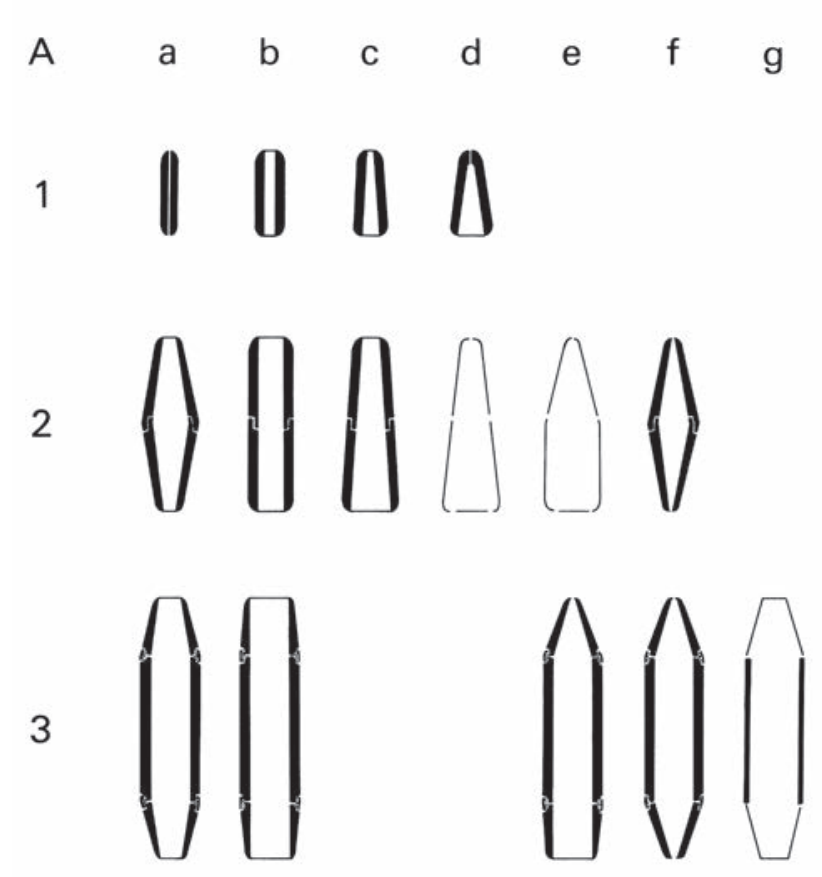

Fig. 17: Typologie des embarcations polygonales, avec des bouchains (en noir) constitués par un (1), deux (2) ou trois (3) éléments façonnés par réduction dans un tronc (Arnold 1992, vol. 2, p. 74).

les embarcations monoxyles, les constructions «à structure monoxyle-assemblée» et celles assemblées, c'est-à-dire dont les bordages sont exclusivement constitués de planches, à l'image de l'épave 8 de Lyon/Parc Saint-Georges. Il souligne que l'« architecture monoxyle assemblée» repose sur une structure monoxyle éclatée en un ensemble de pièces peu nombreuses, dont les plus importantes sont les bordés monoxyles de transition qui vont ceinturer la sole (Rieth 2006, p. 49). 


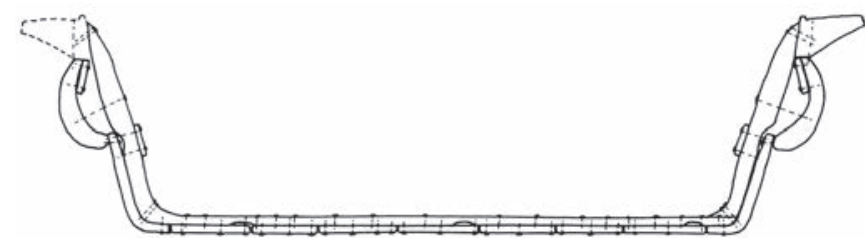

Fig. 18: Coupe transversale du chaland gallo-romain de Xanten-Lüttingen (Obladen-Kauder 1995, fig. 54).

\section{LES EMBARCATIONS CONSTITUÉES PAR UN PATCHWORK D'ÉLÉMENTS MONOXYLES ET PIROGUES ABOUTÉES}

En l'absence d'arbres aux dimensions suffisantes, on observe parfois la présence de coques constituées par un assemblage de pièces hétéroclites obtenues, pour certaines d'entre elles, voire la totalité, par réduction. Il en est ainsi des grandes embarcations markaba des pêcheurs Kotoko (rive sud-ouest du lac Tchad), mais également de celles de Niafounké sur le fleuve Niger, assemblées au moyen de grosses ligatures (Bidault 1945, p. 270-271, pl. p. 256-257)

Ces embarcations, constituées par un patchwork d'éléments monoxyles, correspondent à une phase de transition induite par l'absence d'une matière première aux dimensions suffisantes pour l'obtention de grandes pièces longitudinales par soustraction ou/et l'absence généralisée de grandes scies de long. Le concept de leur réalisation reste toutefois encore très proche de celui mis en œuvre pour la fabrication des pirogues monoxyles.

Autre cas particulier: les esquifs utilisés au Mali, sur le fleuve Niger, sont parfois constitués par l'assemblage de deux demi-pirogues coupées transversalement; ces dernières sont généralement constituées par un assemblage de petites planches ligaturées (aujourd'hui clouées). Ces deux éléments sont réunis par une ligature massive, actuellement encore présente sur le flanc de quelques spécimens (fig. 19; Pitot, Daget 1948, Hagemann 1983); une technique en voie de disparition remplacée par un clouage intensif des pièces assemblées par une longue enture oblique à mi-bois. Ces esquifs sont appelés pirogues de Djenné par A. Pitot et J. Daget (1948, p. 5, fig. p. 14-15). On peut les classer dans les pirogues aboutées, c'est-à-dire constituées de deux éléments assemblés bout à bout au moyen d'une entablure droite, à plats joints, en biseau, à mi-bois, etc. (Gerner 1995); en revanche, elles ne peuvent être classées dans les embarcations polygonales.

\section{PLANCHE(S) AXIALE(S)}

Bien que ne faisant pas directement partie des embarcations basées sur le concept de la soustraction, il nous faut néanmoins évoquer celles basées sur une ou deux planches axiales, à l'image des bateaux de l'âge du Bronze de North Ferriby (Wright 1990, fig. 3.10 et 5.6) ${ }^{16}$. Les tenons mortaisés réservés à la surface des planches montrent que ces éléments sont, en fait,

16. On peut développer un raisonnement similaire pour les embarcations gallo-romaines ou celto-romaines de la façade atlantique, telles celles de Blackfriars 1, St. Peter Port 1 ou Barland's Farm, qui ne font donc pas partie des issus d'un façonnage par réduction dans une épaisse pièce de bois. On notera surtout l'absence d'une pièce axiale avec une coupe transversale en forme de $U$ ou de deux pièces longitudinales avec une coupe en $\mathrm{L}$, donc l'absence d'un profilé, d'une cornière en $\mathrm{U}$ ou en $\mathrm{L}$, avec tous les avantages que ces éléments apportent à la rigidité longitudinale pour un poids propre minimal.

La jonction entre une planche axiale et les bordages des flancs au niveau des extrémités, en particulier ceux situés en contact avec la planche axiale, nécessite l'utilisation de planches vrillées, par exemple en assouplissant le bois en le chauffant, ou alors en taillant cette forme tridimensionnelle complexe par soustraction dans une volumineuse pièce de bois. Dans ce cas précis, on est indubitablement en présence d'une solution technique basée sur la soustraction et non plus d'un concept de construction basé sur la soustraction; l'identification de ce dernier pouvant ainsi être étayé par la présence de profilés ligneux.

Avec ces critères, le bateau de l'âge du Bronze de Douvres peut être classé dans les embarcations polygonales (Clark (éd.) 2004, fig. 5.1), donc des embarcations basées sur le concept de la soustraction, même si les deux grandes planches axiales (constituant quasiment la totalité du fond plat), avec leurs nervures longitudinales et leurs grosses réserves mortaisées, souligneraient plutôt une appartenance de ce bateau au concept des embarcations à planche(s) axiale(s).

\section{ESQUIFS ET EMBARCATIONS OBTENUS PAR SOUSTRACTION}

Pirogues monoxyles, surélevées, expansées, à balancier(s), couplées, embarcations à base polygonale ou en patchwork sont caractérisées par le recours à l'évidage d'une ou plusieurs masses de bois, donc au principe de la soustraction de matière.

En raison de la simplicité de cette technique, les monoxyles constituent quasiment un passage obligé menant ensuite vers des formes plus élaborées. Il n'est donc pas étonnant que le monoxyle et ses dérivés élaborés aient été observés en de multiples occasions, dans l'espace et dans le temps, sur toute la planète où poussent des arbres. Avec une maîtrise relative du feu, l'outillage peut, à l'origine, être limité à l'utilisation de racloirs de fortune: une perche biseautée, un gros coquillage, un chopping-tool (Hariot 1590, pl. XII). Le plus gros problème à surmonter a certainement été d'ordre psychologique: s'attaquer à un géant de la forêt. Et aujourd'hui encore (ou plutôt hier), de nombreuses cérémonies entourent l'abattage d'un arbre afin que l'esprit de ce dernier ne se venge pas par la suite sur le pêcheur ou les utilisateurs de l'esquif ${ }^{17}$.

Par la suite, les charpentiers ont essayé de se libérer des contraintes dimensionnelles issues de la matière, c'est-à-dire de la géométrie cylindrique des arbres et de leurs dimensions, de rendre parfois plus compacte la forme effilée des pirogues, et de trouver des solutions de substitution pour remplacer la forme arrondie des extrémités. Les solutions, les voies sont

embarcations basées sur le concept de la soustraction de matière, contrairement à celles, contemporaines, de l'espace fluvial rhénan et rhodanien.

17. Voir par exemple Kunze 1968, pour le Mondsee, en Autriche; Archaimbault 1972, pour les pirogues de course du Laos; Ivanoff 1999, p. 6-7 et 29-32, pour les Moken, sur la côte sud du Myanmar; Stewart 1995, p. 37-39, pour les Haïdas de la Colombie britannique; etc. 

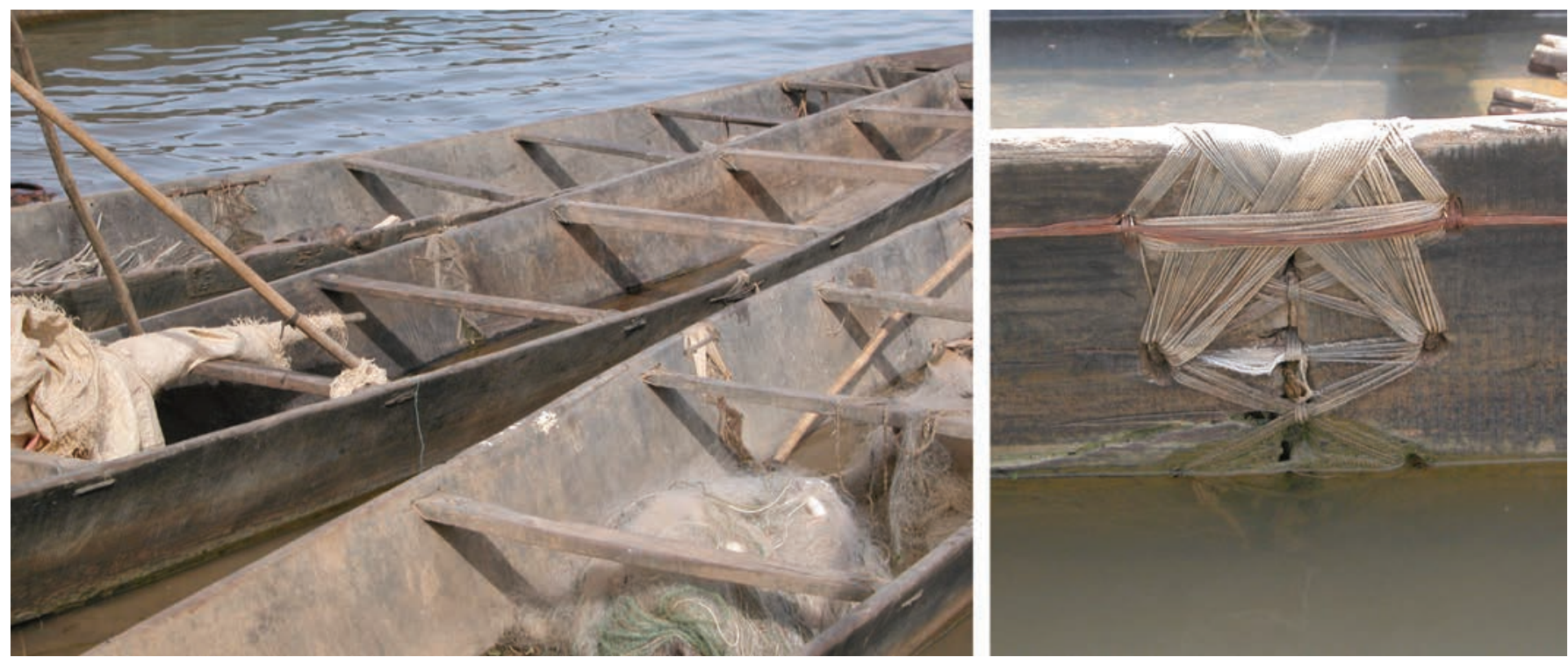

Fig. 19: Demi-pirogues dont les flancs sont encore assemblés par une ligature majeure située au milieu de l'esquif. Le fond, la sole, est déjà formé par une seule entité constituée de pièces assemblées par clouage (clichés B. Arnold ; petit village Bozo sur les rives du Niger, en face de Ségou, Mali ; janvier 2004).

nombreuses; la construction par encorbellement inverse en est une, originale.

La disparition de la matière première en raison du déboisement généralisé auquel est soumise notre planète va inévitablement induire le développement de nouvelles solutions pour les populations dépendant des constructions de monoxyles. Adaptations dont nous trouverons parfois des parallèles dans les vestiges du passé. Il n'en reste pas moins que, si l'aspect matériel pourra être analysé dans le futur au moyen de pièces muséographiques ou de vestiges archéologiques, les apports culturels et cultuels seront perdus à tout jamais si le fil de la tradition orale n'est pas sauvegardé (ou documenté) aujourd'hui, bribe après bribe, chacun y apportant l'empreinte de ses propres connaissances (Archaimbault 1972).

Nous avons donc subdivisé les embarcations basées sur le concept de la soustraction en quatre groupes (fig. 20). On restera toutefois attentif au fait que cette technique peut parfois être appliquée dans d'autres constructions afin de résoudre ponctuellement un problème spécifique, à l'image de la fixation des extrémités des virures des bateaux vikings sur l'étrave ou l'étambot; mais l'embarcation ne fait pas pour autant partie du groupe basé sur le concept de la construction par soustraction.

Notre premier groupe, le plus simple, est constitué par une pirogue monoxyle stricto sensu (1), qui correspond à l'une, voire la plus répandue des embarcations utilisées à travers le monde jusqu'au début du $\mathrm{Xx}^{\mathrm{e}}$ siècle.

Le second est caractérisé par l'adjonction d'éléments destinés à faire évoluer le monoxyle, ce dernier constituant l'élément basal et axial de toute la construction (2). Cette base axiale monoxyle peut être exclusivement surélevée par des éléments rapportés (2.1). L'une des tendances que l'on observe parfois est une réduction de cet élément axial, par exemple sous la forme d'une gouttière qui officiera alors comme un patin lors de la mise à l'eau de l'esquif. Cet élément peut se réduire jusqu'à devenir une planche un peu plus épaisse que les autres bordages, puis prendre une section en forme de T. On quitte alors progressivement le concept de la soustraction pour aborder finalement la famille des embarcations à planche(s) axiale(s) puis à quille.
Cette base axiale monoxyle peut également être déformée plastiquement par chauffage du bois, c'est-à-dire expansée (2.2); elle sera usuellement surmontée d'une ou de plusieurs virures.

Dans le cas de la surélévation comme dans l'expansion, la présence d'une base monoxyle permet de résoudre par soustraction l'arrondi des extrémités ou, à l'inverse, le façonnage de formes très pincées. Parmi les solutions destinées à se substituer à cette base monoxyle, on peut relever la technique mise en œuvre sur les patia utilisés sur la côte de l'Orissa, caractérisés par un empilement de planches décalées, technique que nous avons qualifiée de construction par encorbellement inverse (2.5).

Nous avons considéré que l'introduction du balancier (2.3) correspond, dans les faits, à un développement équivalent à celui de la surélévation ou/et à l'expansion, car elle ouvre la possibilité de parcourir les océans, en particulier à l'aide d'une voile, offrant ainsi à l'homme une nouvelle dimension maritime, que le balancier soit simple ou double. Quant à la coque de ces esquifs (monoxyle, surélevée ou plus rarement expansée), elle est souvent trop étroite pour être utilisée de manière indépendante: sans balancier, l'embarcation se couche sur la plage ou chavire en mer.

Nous avons enfin ajouté l'ensemble des pirogues munies de stabilisateurs (2.4), c'est-à-dire d'éléments accolés à un élément axial monoxyle, généralement de faibles dimensions, dont les perspectives évolutives restent limitées.

Le troisième groupe est constitué de monoxyles multiples (3). On compte dans cet ensemble les esquifs constitués par deux éléments généralement reliés par une plateforme, embarcations que nous qualifierons de pirogues couplées (3.1). Les problèmes de stabilité transversale disparaissent quasi totalement. On observe une utilisation fréquente de ce genre d'embarcation comme ferry, pour transporter des charges encombrantes d'une rive à l'autre, mais également pour la colonisation des îles du Pacifique. On constate aussi l'existence d'embarcations constituées par des pirogues juxtaposées (3.2), c'est-à-dire un ensemble de pièces accolées les unes aux autres. Une telle structure dérive alors plutôt du concept du radeau où 
1.1 monoxyles stricts

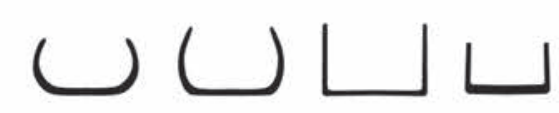

2.1 surélévation simple

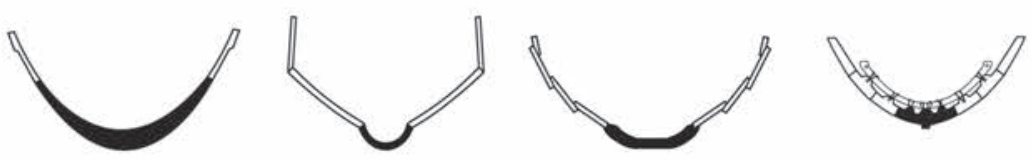

2.2 expansion (généralement complétée par une surélévation)
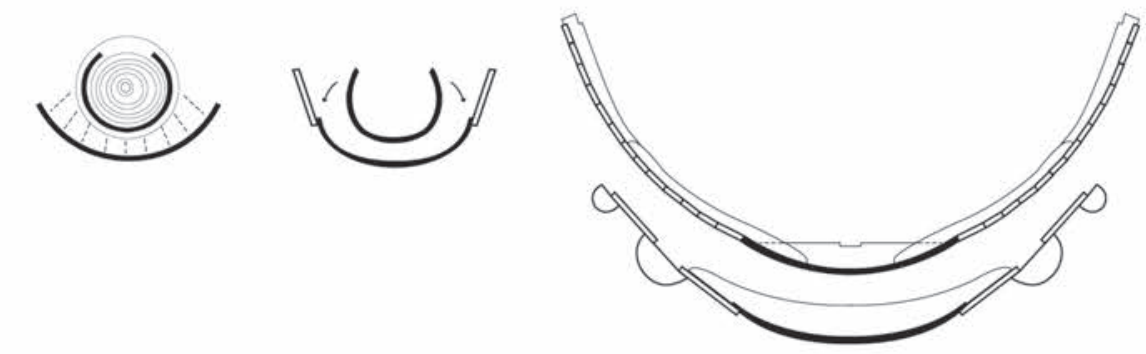

2.3 balancier(s)
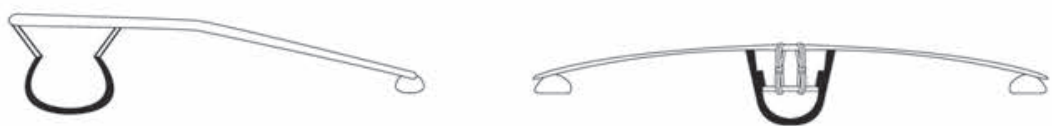

2.4 stabilisateurs

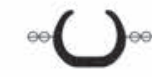

2.5 construction par encorbellement inverse

solutions originales pour remplacer la base monoxyle

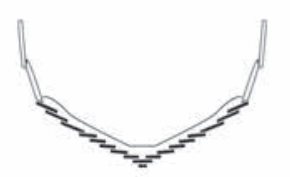

3.1 pirogues couplées

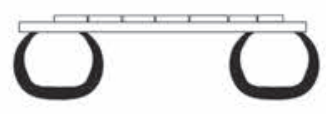

3.2 pièces juxtaposées (concept du radeau)

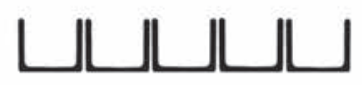

4.1 embarcations polygonales
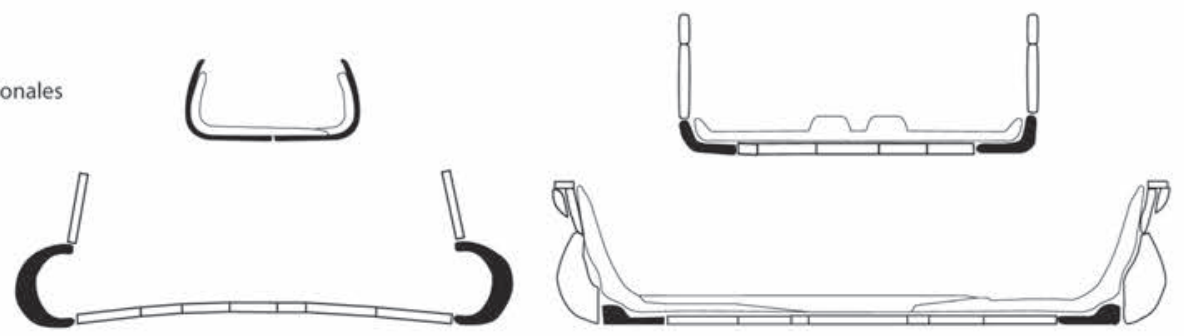

4.2 patchwork d'éléments monoxyles

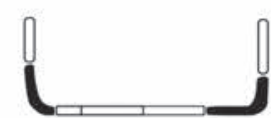

4.3 pirogues aboutées

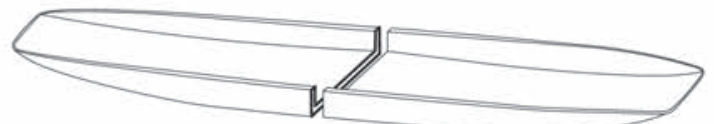

Fig. 20: Typologie des embarcations basées sur le concept de la soustraction. Base des esquifs utilisés pour cette figure: 1.1) vallum (Kapitän 2009), pirogues d'Aegeri, du Chiemsee et du Mondsee (Arnold 1983); 2.1) waka taua (Best 1925), e kuo de Nauru (Haddon et Hornell 1936), Bodenschalenboot-Kahn (Rudolph 1966), pledang de Lamalerap (Horridge 1982); 2.2) expansion de la base du bateau d'Utrecht (Van de Moortel 2003), canot des Boni (Hurault 1970), jukung raksasa (Petersen 2000), bateau d'Utrecht (Van de Moortel 2003); 2.3) pirogue de Goa (Pâris 1841-1842), pirogue de Menado (Hornell 1920); 2.5) patia (McGrail 2003); 3.1) añgula (Kapitän 2009); 3.2) rivière Dunajec (d'après Boczar 1966); 4.1) barque d'Yverdon-2 et chaland de Bevaix (Arnold 1992); mā-däl-pāru (Kapitän 2009), Lyon/Parc Saint-Georges groupe 1 (Guyon, Rieth 2011); 4.2) markaba des Kotoko (reconstitué); 4.3) kole kole ou barque de Djenné (Pitot, Daget 1948) (dessins B. Arnold; DAO M. Arnold). 
la flottabilité est assurée par la réunion de multiples éléments individuels.

Le quatrième groupe, contrairement au deuxième, voit les éléments façonnés par soustraction gagner les côtés de la coque de l'embarcation: on parlera de bateaux à éléments monoxyles assemblés (4). Ces derniers sont dérivés du concept d'une pirogue fendue selon son axe longitudinal. L'embarcation présentera ainsi un fond plus ou moins plat dans le sens transversal, et les éléments du bouchain induiront une forme polygonale au bateau (4.1). Cet éclatement de la forme permettra ainsi de faire varier sa géométrie et ses dimensions.

Nous avons ajouté à ce groupe les quelques embarcations constituées par un patchwork (4.2), un assemblage hétéroclite d'éléments obtenus par soustraction, en particulier au niveau des bouchains. Quant aux esquifs constitués par deux demi-pirogues aboutées (4.3), c'est-à-dire assemblées bout à bout, ils sont à rapprocher de ceux constitués par un patchwork dans une forme initiale.

Nous n'avons donc pas opté pour une classification dichotomique stricte, mais plutôt pour un regroupement d'ensembles qui présentent des particularités spécifiques, observées sur des artefacts dispersés dans l'espace et dans le temps, où c'est plutôt le concept ayant abouti à l'embarcation considérée qui a été mis en relief.

Béat ARNOLD

Archéologue cantonal retraité Laténium, 2068 Hauterive, Suisse beat.arnold47@gmail.com

\section{BIBLIOGRAPHIE}

\section{ANDERSEN S. H.}

1994 New finds of Mesolithic logboats in Denmark, dans C. Westerdahl (ed.), Crossroads in Ancient shipbuilding. Proceedings of the Sixth International Symposium on Boat and Ship Archaeology, Roskilde, 1991, Oxford, Oxbow Books (Oxbow Monograph, 40), p. 1-10.

Archaimbault C.

1972 La course de pirogues au Laos: un complexe culturel, Ascona, Artibus Asiae Publishers (Artibus Asiae, suppl. 29).

ARNOLD B.

1992 Batellerie gallo-romaine sur le lac de Neuchâtel, Saint-Blaise, Editions du Ruau (Archéologie neuchâteloise, 12 et 13).

1995-1996 Pirogues monoxyles d'Europe centrale: construction, typologie, évolution, Neuchâtel, Musée cantonal d'archéologie (Archéologie neuchâteloise, 20 et 21).

1999 Altaripa: archéologie expérimentale et architecture navale gallo-romaine, Neuchâtel, Musée cantonal d'archéologie (Archéologie neuchâteloise, 25).

2006 Archéologie expérimentale: la pirogue néolithique expansée Paris-Bercy 6 et les arts du feu, dans B. Arnold, N. Bauermeister, D. Ramseyer (éd.), Archéologie plurielle. Mélanges offerts à Michel Egloff à l'occasion de son $65^{e}$ anniversaire. Neuchâtel, Musée cantonal d'archéologie (Archéologie neuchâteloise, 34), p. 65-77.

2011 Souvenirs de vacances: le tour du monde en 80 pirogues et quelques embarcations traditionnelles (2000-2010), La Tène (Marin-Epagnier), à compte d'auteur (CD-Rom).

BASCH L.

1987 Le musée imaginaire de la marine antique, Athènes, Institut hellénique pour la préservation de la tradition nautique.

Best E.

1925 The Maori Canoe. An account of various types of vessels used by the Maori of New Zealand in former times. With some description of those of the Isles of the Pacific, and a brief account of the peopling of New Zealand, Wellington, Dominion Museum (Bulletin, 7).

BiDAULT J.

1945 Pirogues et pagaies, Paris, Susse.

Blue L., Kentley E., McGrail S., Mishra U.

1997 The Patia fishing boat of Orissa: a case study in ethnoarchaeology, South Asian Studies, 13, p. 189-207.

\section{Boczar M. L.}

1966 The craft in use at the rivergate of the Dunajec, The Mariner's Mirror, 52.3, p. 211-222.

\section{BRUNNER K.}

1903 Donaugebiet. In: Zur Forschung über alte Schiffstypen auf den Binnengewässern und an den Küsten Deutschlands und der angrenzenden Länder, Correspondenz-Blatt der deutschen Gesellschaft für Anthropologie, Ethnologie und Urgeschichte, 34/1-2, p. 1-13.

Camus A.-G.

$1802 \quad$ Mémoire sur la collection des Grands et Petits Voyages [des de Bry] et sur la collection des voyages de Melchisedech Thevenot, Paris, Baudouin

Christensen A. E.

1977 Ancient boatbuildig - a provisional calssification, dans S. McGrail (ed.), Sources and techniques in boat archaeology, Oxford, BAR (BAR Suppl. Series 29), p. 269-280.

Clark P. (éd.)

2004 The Dover Bronze Age Boat, Swindon, English Heritage.

CoOK J., Furneaux T.

1777 A voyage towards the South Pole, and round the world, performed in his Majesty's ships the Resolution and Adventure, in the years 1771, 1773, 1774, and 1775, Londres, Strahan and Cadell.

Crumlin-Pedersen O.

1972 Skin or wood? A study of the origin of the Scandinavian plankboat, dans O. Hasslöf, H. Henningsen, A. E. Christensen (eds), Ships and shipyards, sailors and fishermen. Introduction to maritime ethnology, Copenhague, Rosenkilde et Bagger, p. 208234

2010 Archaeology and the sea in Scandinavia and Britain. A personal account, Roskilde, Viking Ship Museum (Maritime Culture of the North, 3).

EDWARDS R

1972 Aboriginal bark canoes of the Murray Valley, Adelaide, Rigby (South Australian Museum).

EDYE J.

1834 Description of the various classes of vessels constructed and employed by the natives of the Coasts of Coromandel, Malabar, and the Island of Ceylon, for their coasting navigation, The 
Journal of the Royal Asiatic Society of Great Britain and Ireland, 1, p. 1-14.

ELLMERS D

1973 Kultbarken, Fähren, Fischerboote. Vorgeschichtliche Einbäume in Niedersachsen, Die Kunde, n.s. 24, p. 23-62.

ESKERÖD A.

1956 Early Nordic-Arctic Boats. A survey and some problems, Arctica. Studia ethnographica Upsaliensia, 11, p. 57-87.

\section{Gerner M.}

1995 Les assemblages des ossatures et charpentes en bois, Paris, Eyrolles.

GR ÄF D.

2006 Boat mills in Europe from Early Medieval to Modern Times, Dresde, Landesamt für Archäologie mit Landesmuseum für Vorgeschichte (Bibliotheca Molinlogica, 19).

GRANDSAIGNES T. DE

1906 Les pirogues monoxyles néolithiques du lac Chalain, L'Homme préhistorique, 4, p. 321-326.

Greenhill B.

1976 Archaeology of the boat. A new introductory study, Londres, Black.

Groslier B. P.

1982 L'Inde, dans Le grand atlas de l'architecture mondiale, Encyclopaedia Universalis France, p. 54-63.

GuYON M., Rieth É.

2001 Les chalands gallo-romains du Parc Saint-Georges, dans G. Boetto, P. Pomey, A. Tchernia (éd.), Batellerie gallo-romaine. Pratiques régionales et influences maritimes méditerranéennes, Paris, Edition Errance, Centre Camille Jullian (BiAMA, 9), p. $91-101$.

HARIOT T.

$1590 \quad$ A brife and true report of the new found land of Virginia, Frankfurt am Main, Theodor de Bry (Th. de Bry "Grands Voyages", partie 1); fac-similé (Dover Publication, New York, 1972, avec une introduction de P. Hulton).

Haddon A. C., Hornell J.

1936 Canoes of Oceania. Vol.1. The canoes of Polynesia, Fiji, and Micronesia, Honolulu, Bishop Museum (Special publications, 27).

1937 Canoes of Oceania. Vol.2. The canoes of Melanesia, Queensland, and New Guinea, Honolulu, Bishop Museum (Special publications, 28).

1938 Canoes of Oceania. Vol.3. Definition of terms, general survey, and conclusions, Honolulu, Bishop Museum (Special publications, 29).

Hagemann A.

1983 Bootsbau in Mali, Baessler-Archiv, Beiträge zur Völkerkunde, NF $31 / 1$, p. 57-135.

HaLldin G.

1950 Bilder av primitiva farkoster m. m. sammanställda för studiet av de skandinaviska hällristningarnas skeppsbilder, Sjöhistorisk Årsbok, p. 11-96.

HARDGRAVE R. L.

2004 A portrait of the Hindus. Balthazar Solvyns and the European image of India 1760-1824, Oxford, University Press.

\section{HORNELL J}

1920 The Outrigger Canoes of Indonesia, Madras Fisheries Bulletin, 12 , p. 43-114.

1946 Water transport. Origins and early evolution, Cambridge, University Press.
HorRidge A. G.

1982 The lashed-lug boat of the eastern archipelagoes, the Alcina MS and the Lomblen whaling boat, Greenwich, National Maritime Museum (Maritime monographs and reports, 54).

Hurault J.

1970 Africains de Guyane. La vie matérielle et l'art des Noirs Réfugiés de Guyane, La Haye et Paris, Éditions Mouton.

\section{ITKONEN T. I.}

1941 Suomen ruuhet. 1-, 2-, 3-ja monipuiset sekä lautaruuhet kivikaudesta vuoteen 1940 (mit deutschen Referaten), Forssa, Suomen Muinaismuistoyhdistys (Kansatieteellinen Arkisto, $5 / 1)$.

IVANOFF J.

1999 The Moken boat: symbolic technology, Bangkok, White Lotus.

KAPITÄN G.

2009 Records of traditional watercraft from South and West Sri Lanka, Oxford, Archeopress (BAR Int. Series, 1931).

KunZE W.

1968 Der Mondseer Einbaum, Jahrbuch des Oberösterreichischen Musealvereines, 113, p. 173-202.

KRÖGER L.

2009 Die Einbäume des Maingebietes. Fährwesen im Mittelalter, Bamberg, Otto-Friedrich-Universität (Magisterarbeit).

2011 Einbäume des Maingebietes. Fähren als verbindendes Element eines mittelalterlichen und frühneuzeitlichen Wegesystems, Siedlungs- und Küsenforschung im südlichen Nordseegebiet, 34 , p. $115-128$.

LÄCHLER P., Wirz H.

1962 Die Schiffe der Völker, Olten und Freiburg in Breisgau, Walter.

LE RoY J. D.

1777 La marine des anciens peuples, expliquée et considérée par rapport aux lumières qu'on en peut tirer pour perfectionner la Marine moderne. Avec des figures représentant les vaisseaux de guerre de ces peuples, Paris, Nyon, Stoupe.

LINDBERG M.

2012 The Byslätt Bronze Age Boat. A Swedish Bark Canoe, University of Southern Denmark (master thesis).

LOTHROP S. K.

1932 Aboriginal navigation off the West coast of South America, Journal of the Royal Anthropological Institute, 62, p. 229-256.

LOUPPE D. (éd.)

2008 Bois d'œuvre, 1, Leiden, Backhuys (Plant Resources of Tropical Africa/Ressources végétales de l'Afrique tropicale, 7).

McGrail S

1985 Towards a classification of water transport, World Archaeology, 16/3, p. 289-303.

1987 Ancien boats in N.W. Europe. The archaeology of water transport to AD 1500, Londres- New York, Longman.

2003 Boats of South Asia, Londres, Routledge Curzon.

Medas $\mathrm{S}$.

1993 Imbarcazioni e navigazione preistorica nel Mediterraneo, Bollettino di Archeologia Subacquea, 1, n 0, p. 103-147.

Mills G. G.

1994 Malawi - Villages de pêcheurs et pépinières forestières, Unasylva, Revue internationale des forêts et des industries forestières, 45, 2, $\mathrm{n}^{\circ} 177$ (http://www.fao.org/docrep/t2230F/ t2230f0b.htm).

\section{Moortel A. VAn DE}

2003 A new look at the Utrecht ship, dans C. Beltrame (ed.), Boats, Ships and Shipyards. Proceedings of the Ninth International 
Symposium on Boat and Ship Archaeology, Venice, 2000, Oxford, Oxbow Books, p. 183-189.

\section{OBLADEN-KaUder J.}

1995 Bodendenkmalpflege am Beispiel der niederrheinischen Kiesgruben. Aspekte rezenter fluvialer Archäologie, Archäologische Informationen aus Baden-Württemberg, 33, p. 85-91.

PÂRIS F.-E.

1843 Essai sur la construction navale des peuples extra-européens ou collection des navires et pirogues construits par les habitants de l'Asie, de la Malaisie, du Grand Océan et de l'Amérique, Paris, Arthus Bertrand.

Petersen E.

2000 Jukung-boats from the Barito Basin, Borneo, Roskilde, The Viking Ship Museum.

\section{Pitot A., Daget J.}

1948 Les barques du Moyen Niger, Paris, Gauthier-Villars (Documents d'ethnographie navale, 5).

\section{PoMey P.}

1988 Principes et méthodes de construction en architecture navale antique, dans Navires et commerces de la Méditerranée antique. Hommage à Jean Rougé, Cahiers d'Histoire, 33/3-4, p. 397-412.

1994 Shell conception and skeleton process in Ancient Mediterranean shipbuilding, dans C. Westerdahl (ed.), Crossroads in Ancient shipbuilding. Proceedings of the Sixth International Symposium on Boat and Ship Archaeology, Roskilde, 1991, Oxford, Oxbow Books (Monograph, 40), p. 125-130.

1998 Conception et réalisation des navires dans l'Antiquité méditerranéenne, dans E. Rieth (dir.), Concevoir et construire des navires. De la trière au picoteux, Ramonville Saint-Agne, Editions Erès (Technologies/Idéologie/Pratiques, 13/1), p. 49-72.

2004 Principles and methods of construction in Ancient naval architecture, dans F. M. Hocker, C. A. Ward (eds.), The philosophy of shipbuilding. Conceptual approches to the study of wooden ships, College Station, Texas A\&M University Press, p. 25-36.

2012 A propos des navires de la mer Erythrée: découvertes récentes et nouveaux aspects de la question, Topoi, suppl. 11, p. 111-132.
Poujade J.

1946

La route des Indes et ses navires, Paris, Payot.

RIETH É.

1979 Quelques problèmes récents d'archéologie navale en Europe de l'Ouest et du Nord, Archéologie médiévale, 9, p. 209-222.

1981 La construction navale à fond plat en Europe de l'Ouest, Ethnologie française, 11, 1, p. 47-62, 95.

2006 Archéologie de la batellerie et architecture nautique fluviale, Conflans-Sainte-Honorine, Association des Amis du Musée de la Batellerie (Les Cahiers du Musée de la Batellerie, 56).

RUDOLPH W.

1966 Handbuch der volkstümlichen Boote im östlichen Niederdeutschland, Berlin, Akademie-Verlang (Veröffentlichungen des Instituts für deutsche Volkskunde, 41).

Spranz B. (éd.)

1984 Boote. Technik und Symbolik. Die Schiffahrt in aussereuropäischen Kulturen, catalogue d'exposition, Freiburg im Breisgau, Museum für Völkerkunde.

Stewat H.

1995 Cedar: Tree of life to the Northwest Coast Indians, Seattle, University of Washington Press.

SUDER H.

1930 Vom Einbaum und Floss zum Schiff. Die primitiven Wasserfahrzeuge, Berlin, Mittler (Veröffentlichungen des Instituts für Meereskunde, NF Beiheft 7).

\section{VLIERMAN K.}

$1996 \quad$ Kleine bootjes en middeleeuws scheepshout met constructieetails door, Lelystad, Nederlands Instituut voor scheeps- en onderwaterarcheologie (Flevobericht, 404; Scheepsarcheologie, 2)

WRIGHT E.

1990 The Ferriby boats. Seacraft of the Bronze Age, Londres, New York, Routledge. 